\title{
ON CRYSTAL OPERATORS IN LUSZTIG'S PARAMETRIZATIONS AND STRING CONE DEFINING INEQUALITIES
}

\author{
SHMUEL ZELIKSON \\ LMNO, UMR 6139 du CNRS, Département de Mathématiques, \\ Université de Caen, B.P. 5186, 14032 Caen Cedex, France \\ e-mail:Shmuel.Zelikson@unicaen.fr
}

(Received 14 October 2011; revised 7 March 2012; accepted 15 March 2012; first published online 2 August 2012)

\begin{abstract}
Let $\mathbf{w}_{0}$ be a reduced expression for the longest element of the Weyl group, adapted to a quiver of type $A_{n}$. We compare Lusztig's and Kashiwara's (string) parametrizations on canonical basis associated with $\mathbf{w}_{0}$. Crystal operators act in a finite number of patterns in Lusztig's parametrization, which may be seen as vectors. We show that this set gives the system of defining inequalities of the string cone constructed by Gleizer and Postnikov (O. Gleizer and A Postnikov, LittlewoodRichardson coefficients via Yang-Baxter equation, IMRN 14 (2000) 741-774). We use combinatorics of the Auslander-Reiten quivers, and as a by-product we get an alternative enumeration of a set of inequalities defining the string cone based on hammocks.
\end{abstract}

AMS Mathematics Subject Classification. 17B37, 05E10, 16G70.

1. Introduction. Let $U_{q}(\mathfrak{g})$ be the quantized enveloping algebra corresponding to a Dynkin diagram $D$ of type $A_{n}, D_{n}, E_{n}$, defined over $\mathbb{C}(q), q$ being an indeterminate. There is a braid group action on $U_{q}(\mathfrak{g})$ which enables to construct Poincaré-BirkhoffWitt (PBW) bases [15] of the positive part $U_{q}\left(\mathfrak{n}^{+}\right)$of $U_{q}(\mathfrak{g})$. Such a basis $\mathcal{P}_{\mathbf{w}_{0}}$ depends on the choice of a reduced expression $\mathbf{w}_{0}$ of the longest element $w_{0}$ of the Weyl group $W$ associated with $D$. However, it was observed by Lusztig that the $\mathbb{Z}\left[q^{-1}\right]$-module $\mathcal{L}$ generated by $\mathcal{P}_{\mathbf{w}_{0}}$ is independent of $\mathbf{w}_{0}$. Furthermore, the image of $\mathcal{P}_{\mathbf{w}_{0}}$ under the projection $\pi: \mathcal{L} \longrightarrow \mathcal{L} / q^{-1} \mathcal{L}$ is a $\mathbb{Z}$-basis $B$ of $\mathcal{L} / q^{-1} \mathcal{L}$, which is again independent of $\mathbf{w}_{0}$. There is a unique basis of $\mathcal{L}$, which is invariant under the $\mathbb{C}$-algebra involution of $U_{q}\left(\mathfrak{n}^{+}\right)$preserving the generators of $U_{q}\left(\mathfrak{n}^{+}\right)$, and sending $q$ to $q^{-1}$, and whose image under $\pi$ is $B$. This is the canonical basis $\mathcal{B}_{\text {can }}$ of Lusztig and Kashiwara [15, 12]. This basis is in one-to-one correspondence with any $\mathrm{PBW}$ basis $\mathcal{P}_{\mathbf{w}_{0}}$, yet is independent of the choice of $\mathbf{w}_{0}$. It has with many remarkable properties. The basis $\mathcal{B}_{\text {can }}$ is, however, difficult to compute for arbitrary Dynkin diagrams.

Let $I$ denote the set of vertices of $D$. Kashiwara introduced crystal operators $\tilde{e}_{i}, \tilde{f}_{i}, i \in I$ on $U_{q}\left(\mathfrak{n}^{+}\right)$. These allow to construct the crystal graph $B(\infty)$ which serves as a combinatorial skeleton of $\mathcal{B}_{c a n}$. Its vertices are the elements of $B$, and its edges are induced by the action of crystal operators on $\mathcal{B}_{\text {can }}$. The crystal limit $b \mapsto b \bmod q^{-1} L$ establishes a one-to-one correspondence between $\mathcal{B}_{c a n}$ and vertices of $B(\infty)$, which allows to extract important combinatorial information from $\mathcal{B}_{\text {can }}$ to the level of $B(\infty)$. The crystal graph $B(\infty)$ may be defined by purely combinatorial means, and provides important data for the study of finite-dimensional representations of $U_{q}(\mathfrak{g})$. 
The crystal limit also establishes a one-to-one correspondence between members of a basis $\mathcal{P}_{\mathbf{w}_{0}}$ and the vertices of $B(\infty)$. A PBW monomial is defined by an $N$-tuple of positive integers, where $N$ is the number of positive roots in the root system associated to $D$. One thus gets an indexation of $B$ by $\mathbb{N}^{N}$. This is the Lusztig parametrization with respect to $\mathbf{w}_{0}$.

Its advantage lies in the simple indexing set for $B$. The action of a crystal operator $\tilde{e}_{i}$ is easy to describe when the reduced expression $\mathbf{w}_{0}$ starts with the simple reflection $s_{i}$. It is however difficult to give, for a fixed $\mathbf{w}_{0}$, the action of all the operators $\tilde{e}_{i}, i \in I$ in the same time, due to the complexity of the passage formulas [4] between different Lusztig parametrizations. This was done by Reineke [18] for reduced expressions $\mathbf{w}_{0}$ adapted to quivers $Q$ of $A D E$ type verifying a particular homological condition ( $L$ ) (detailed in Section 2). The Hall algebra construction [20] of $U_{q}\left(\mathfrak{n}^{+}\right)$allows Reineke to study the crystal operators $\tilde{e}_{i}, i \in I$ using the representation theory of finite-dimensional algebras.

Kashiwara showed [13] that given $\mathbf{w}_{0}$, there is an elementary construction of $B(\infty)$ depending on $\mathbf{w}_{0}$, known as Kashiwara's embedding. The vertices $B$ of $B(\infty)$ are indexed a set $\mathcal{S}_{\mathbf{w}_{0}}$ of specific $N$-tuples of integers, known as string parameters. The action of crystal operators on $B$ is easy to describe, as it depends only on the Cartan matrix of $D$. However, it is a complex problem to describe the parameter set $\mathcal{S}_{\mathbf{w}_{0}}$. It is the set of integer points of a polyhedral cone $\mathcal{C}_{\mathbf{w}_{0}}[\mathbf{1 4}, \mathbf{4}]$. A system of inequalities defining $\mathcal{C}_{\mathbf{w}_{0}}$ was given by Littelmann [14] for particular reduced expressions with a good structure. Such a set of inequalities, for arbitrary $\mathbf{w}_{0}$, was given by Gleizer and Postnikov [10] in $A_{n}$ case, and Berenstein and Zelevinsky [4] for all finite Dynkin types.

Any cone inequality may be seen as $\mathbf{a} \cdot \mathbf{x} \geq 0$ where $\mathbf{a} \in \mathbb{R}^{N}$ is a vector orthogonal to the hyperplane of the inequality. Thus, a polyhedral cone may be seen as being defined by a finite set of vectors. The methods of [10] and [4] construct respectively sets of vectors $K_{\mathbf{w}_{0}}^{G P}$ and $K_{\mathbf{w}_{0}}^{B Z}$ with integer coordinates, defining $\mathcal{C}_{\mathbf{w}_{0}}$. A move associated to a crystal operator $\tilde{e}_{i}$ in a given parametrization is a vector $\mathbf{v}$ appearing as the difference between $\mathbf{t}$ and $\tilde{e}_{i} \mathbf{t}$ for some $N$-tuple $\mathbf{t}$. We shall denote by $L_{\mathbf{w}_{0}}$ the set of all possible moves, for all $\tilde{e}_{i}, i \in I$, in the Lusztig parametrization with respect to $\mathbf{w}_{0}$. Reineke's construction allows, for the reduced expressions $\mathbf{w}_{0}$ for which it is valid, to describe the set $L_{\mathbf{w}_{0}}$ in terms of the Auslander-Reiten quiver $\Gamma_{Q}$ of $Q$.

The main result of this paper (Theorem 2.4) is that for $\mathbf{w}_{0}$ adapted to a quiver $Q$ of type $A_{n}$, one has $K_{\mathbf{w}_{0}}^{G P}=L_{\mathbf{w}_{0}}$. Thus, the problem of constructing the cone $\mathcal{C}_{\mathbf{w}_{0}}$ seems to be the the same as the one of describing action of operators $\tilde{e}_{i}$ in the Lusztig parametrization for $\mathbf{w}_{0}$. We conjecture that the set of Lusztig moves $L_{\mathbf{w}_{0}}$ defines $\mathcal{C}_{\mathbf{w}_{0}}$ for reduced expression adapted to quivers $Q$ of $A D E$ type, under the assumption that condition $(L)$ required by Reineke on $Q$ is verified. We give in the last section a $D_{n}$ example.

A by-product of the main theorem is that the Auslander-Reiten quivers allow to compute a set of defining inequalities of $\mathcal{C}_{\mathbf{w}_{0}}$. The combinatorics involved is that of hammocks, introduced by Brenner [6]. This provides an alternative to methods given in $[10,4]$.

We would like to thank Bernard Leclerc for many helpful remarks.

2. Combinatorial models of the canonical basis. Let $\Phi$ be the root system corresponding to the Dynkin diagram $D$ and (, ) the Cartan scalar product over $\mathbb{R} \Phi$. We shall denote by $\alpha_{i}, i \in I$ the set of simple roots, $\omega_{i}, i \in I$ the set of fundamental 
weights and by $s_{i}, i \in I$ the simple reflections inside $W$. We shall fix all through this section, a reduced expression $\mathbf{w}_{0}=s_{i_{1}} s_{i_{2}} \cdots s_{i_{N}}$ of the longest element $w_{0}$ of $W$. This expression induces the reflection ordering $\preccurlyeq_{\mathbf{w}_{0}}$ on the set of positive roots $\Phi^{+}$, a total ordering given by $\beta_{1}=\alpha_{i_{1}}, \beta_{2}=s_{i_{1}}\left(\alpha_{i_{2}}\right), \ldots, \beta_{N}=s_{i_{1}} s_{i_{2}} \cdots s_{i_{N-1}}\left(\alpha_{i_{N}}\right)$.

We shall use here the conventions of [4], concerning the quantized enveloping algebra $U_{q}(\mathfrak{g})$ associated to $D$. It is generated by the set $e_{i}, f_{i}, k_{i}^{ \pm 1}, i \in I$ subject to relations derived from the Cartan matrix $C$. The reader may find details of the defining relations in [4, Section 3.1]. We recall in particular that $[n]_{q}$ ! denotes the $q$-factorial of $n$, and that the $n$th divided power of an element $x \in U_{q}(\mathfrak{g})$ is given by $x^{(n)}=x^{n} /[n]_{q}$ !.

The positive part $U_{q}\left(\mathfrak{n}^{+}\right)$is the subalgebra generated by the $e_{i}, i \in I$. It admits a grading by $Q^{+}=\bigoplus \mathbb{N} \alpha_{i}$ obtained by putting $\operatorname{deg}\left(e_{i}\right)=\alpha_{i}$. Given an arbitrary $\gamma \in Q^{+}$, the weight space $U_{q}\left(\mathfrak{n}^{+}\right)_{\gamma}$ is the $\mathbb{C}(q)$-vector space of elements of degree $\gamma$. All weight spaces of $U_{q}\left(\mathfrak{n}^{+}\right)$are finite-dimensional.

The braid group acts on $U_{q}(\mathfrak{g})$ by automorphisms $T_{i}, i \in I$ (noted $T_{i,-1}^{\prime}$ in [16, part VI]). We refer again to [4, Section 3]for a detailed definition. For every $k=1,2, \ldots, N, E_{\beta_{k}}:=T_{i_{1}} T_{i_{2}} \cdots T_{i_{k-1}}\left(E_{i_{k}}\right)$ is an element of $U_{q}\left(\mathfrak{n}^{+}\right)$of weight $\beta_{k}$. Any given $N$-tuple $\mathbf{t}=\left(t_{1}, \ldots, t_{N}\right)$ of positive integers defines the PBW monomial

$$
p_{\mathbf{w}_{0}}(\mathbf{t}):=E_{\beta_{1}}^{\left(t_{1}\right)} E_{\beta_{2}}^{\left(t_{2}\right)} \ldots E_{\beta_{N}}^{\left(t_{N}\right)}
$$

The set of all such monomials, $\mathcal{P}_{\mathbf{w}_{0}}:=\left\{p_{\mathbf{w}_{0}}(\mathbf{t}) \mid \mathbf{t} \in \mathbb{N}^{N}\right\}$, forms the $P B W$-basis of $U_{q}\left(\mathfrak{n}^{+}\right)$ associated to the reduced expression $\mathbf{w}_{0}$.

THEOREM 2.1 ([15]). For every monomial $p_{\mathbf{w}_{0}}(\mathbf{t})$, there is one and only one $b \in \mathcal{B}_{\text {can }}$ such that $b=p_{\mathbf{w}_{0}}(\mathbf{t}) \bmod q^{-1} \mathcal{L}$.

The crystal operators $\tilde{e}_{i}, \tilde{f}_{i}, i \in I$ were introduced by Kashiwara [12] for the negative part $U_{q}\left(\mathfrak{n}^{-}\right)$of $U_{q}(\mathfrak{g})$. As $U_{q}\left(\mathfrak{n}^{-}\right)$and $U_{q}\left(\mathfrak{n}^{+}\right)$are isomorphic as algebras, this construction may be carried over to $U_{q}\left(\mathfrak{n}^{+}\right)$.

Given $i \in I$, there is a locally nilpotent action $\theta_{i}$ over $U_{q}\left(\mathfrak{n}^{+}\right)$defined by

$$
\theta_{i}(1)=0, \quad \forall x \in U_{q}\left(\mathfrak{n}^{+}\right): \theta_{i}\left(e_{j} x\right)=q^{\left(\alpha_{i}, \alpha_{j}\right)} e_{j} \theta_{i}(x)+\delta_{i, j} x
$$

One has $U_{q}\left(\mathfrak{n}^{+}\right)=\bigoplus_{n \in \mathbb{N}} e_{i}^{(n)} \operatorname{ker} \theta_{i}$, and $\operatorname{ker} \theta_{i}$ is compatible with the weight graduation of $U_{q}\left(\mathfrak{n}^{+}\right)$. One chooses a weight vector basis $\Xi_{i}$ of $\operatorname{ker} \theta_{i}$, and defines for each $\mathbf{v} \in \Xi_{i}$ and any $n \in \mathbb{N}$ :

$$
\begin{aligned}
& \tilde{e}_{i}\left(e_{i}^{(n)} v\right):=e_{i}^{(n+1)} v, \\
& \tilde{f}_{i}\left(e_{i}^{(n)} v\right):= \begin{cases}e_{i}^{(n-1)} v & \text { if } n \geq 1 \\
0 & \text { if } n=0\end{cases}
\end{aligned}
$$

This leads to well-defined operators $\tilde{e}_{i}, \tilde{f}_{i}$ over $U_{q}\left(\mathfrak{n}^{+}\right)$, which do not depend on the initial choice of $\Xi_{i}$.

The crystal operators $\tilde{e}_{i}, \tilde{f}_{i}, i \in I$ preserve $\mathcal{L}$, and hence induce an action over $\mathcal{L} / q^{-1} \mathcal{L}$. A key feature of the canonical basis is its good behaviour under this action. For any $b \in \mathcal{B}_{\text {can }}, \tilde{e}_{i} b=b^{\prime} \bmod q^{-1} \mathcal{L}$, and $\tilde{f}_{i} b$ is either $0 \bmod q^{-1} \mathcal{L}$ or $\tilde{f}_{i} b=b^{\prime \prime}$ $\bmod q^{-1} \mathcal{L}$, where $b^{\prime}, b^{\prime \prime}$ are other elements of $\mathcal{B}_{\text {can }}$. We see that the image $B$ of $\mathcal{B}_{\text {can }}$ 
inside $\mathcal{L} / q^{-1} \mathcal{L}$ becomes endowed with a structure of a coloured graph, the arrows being valuated by the operators $\tilde{e}_{i}, \tilde{f}_{i}, i \in I$. This is the crystal graph $B(\infty)$ of Kashiwara [12].

Our discussion in the Introduction and Theorem 2.1 above lead to a one-toone correspondence $\varphi_{\mathbf{w}_{0}}: \mathbb{N}^{N} \longrightarrow B, \quad \mathbf{t} \mapsto \mathrm{p}_{\mathbf{w}_{0}}(\mathbf{t}) \bmod q^{-1} \mathcal{L}$. This is the Lusztig parametrization with respect to $\mathbf{w}_{0}$. Under this identification, we may consider the crystal operators $\tilde{e}_{i}, \tilde{f}_{i}$ as acting on $\mathbb{N}^{N}$. We shall call a vector $\mathbf{l} \in \mathbb{Z}^{N}$ the Lusztig move of type $i$ with respect to $\mathbf{w}_{0}$ if there exists $\mathbf{t} \in \mathbb{N}^{N}$ such that $\tilde{e}_{i} \mathbf{t}=\mathbf{t}+\mathbf{l}$. Recall $L_{\mathbf{w}_{0}}$ denotes the set of all possible Lusztig moves for all types $i \in I$.

ExAmple. Consider $A_{2}$ case, $\mathbf{w}_{0}=s_{1} s_{2} s_{1}$. For a given $\mathbf{t}=\left(t_{1}, t_{2}, t_{3}\right)$ one has:

$$
\begin{aligned}
& \tilde{e}_{1}\left(t_{1}, t_{2}, t_{3}\right)=\left(t_{1}+1, t_{2}, t_{3}\right), \\
& \tilde{e}_{2}\left(t_{1}, t_{2}, t_{3}\right)= \begin{cases}\left(t_{1}-1, t_{2}+1, t_{3}\right) & \text { if } t_{1}>t_{3} \\
\left(t_{1}, t_{2}, t_{3}+1\right) & \text { if } t_{1} \leq t_{3}\end{cases}
\end{aligned}
$$

One sees that there is only one Lusztig move of type $1, \mathbf{l}_{1}=(1,0,0)$ and two Lusztig moves of type $2, \mathbf{l}_{2}=(-1,1,0), \mathbf{l}_{3}=(0,0,1)$. We get $L_{\mathbf{w}_{0}}=\{(1,0,0),(-1,1,0),(0,0,1)\}$.

Let $Q$ be a fixed quiver obtained by orienting the Dynkin diagram $D$. Following [5], we call a vertex $i$ of $Q$ a sink if there are only arrows entering it. We denote in that case by $s_{i} Q$ the quiver obtained by reversing the arrows whose end is $i$, into arrows with exiting $i$, thus transforming $i$ into a source. A reduced expression $\mathbf{w}_{0}=s_{i_{1}} s_{i_{2}} \cdots s_{i_{N}}$ is adapted to $\mathrm{Q}$ if and only if $i_{1}$ is a sink of $Q, i_{2}$ is a sink of $s_{i_{1}} Q, i_{3}$ is a sink of $s_{i_{2}} s_{i_{1}} Q$ and so on. Such an expression always exists for a given $Q$.

Let us denote by $\mathbb{C} Q$ the path algebra of $Q$ over $\mathbb{C}$. The category mod $\mathbb{C} Q$ of finite-dimensional left modules has simple objects $S_{i}$ which are indexed by $I$. We shall say, following Reineke [18], that the quiver $Q$ verifies condition $(L)$ if for every indecomposable module $X \in \bmod \mathbb{C} Q$, and every $i \in I$, one has $\operatorname{dim} \operatorname{Hom}\left(X, S_{i}\right) \leq 1$. This condition is verified for any quiver of type $A_{n}$, and at least one quiver each of types $D_{n}, E_{6}, E_{7}$ [18, Appendix]. Under this condition, the action of crystal operators in the Lusztig parametrization with respect to $\mathbf{w}_{0}$ adapted to $Q$ may be described in terms of the category mod $\mathbb{C} Q$, and the set $L_{\mathbf{w}_{0}}$ may be obtained [18]. We postpone the details to Section 3.

We continue to fix the same $\mathbf{w}_{0}=s_{i_{1}}, \ldots s_{i_{N}}$. We refer the reader to Kashiwara [13] and [11, Chapter 5] for details on crystal theory. Kashiwara's elementary construction of $B(\infty)$ uses the Cartan matrix $C=\left(c_{i, j}\right)$ in order to define operators $\tilde{e}_{i}, \tilde{f}_{i}, i \in I$ acting over $\mathbb{N}^{N}$ as below $[11,5.2 .5,6.1 .15]$ :

Fix $\mathbf{a}=\left(a_{1}, \ldots, a_{N}\right) \in \mathbb{N}^{N}$. For $k=1,2, \ldots N$, define $r_{k}:=a_{k}+\sum_{1 \leq j<k} c_{i_{j}, i_{k}} a_{j}$. Given $i \in I$, consider $\xi_{i}=\max _{i_{k}=i} r_{k}$. Let $k_{1}$ be the first position where this maximum is attained, $k_{2}$ is the last. Then

$$
\begin{aligned}
& \tilde{e}_{i}(\mathbf{a})=\left(a_{1}, \ldots, a_{k_{2}-1}, a_{k_{2}}+1, a_{k_{2}+1}, \ldots, a_{N}\right) . \\
& \tilde{f}_{i}(\mathbf{a})=\left\{\begin{array}{cc}
\left(a_{1}, \ldots, a_{k_{1}-1}, a_{k_{1}}-1, a_{k_{1}+1}, \ldots, a_{N}\right) & \text { if } a_{k_{1}} \geq 1 \\
0 & \text { if } a_{k_{1}}=0
\end{array} .\right.
\end{aligned}
$$


THEOREM 2.2 (Kashiwara's embedding [12]). Let $\mathcal{N}$ denotes $\mathbb{N}^{N}$ with the action of operators $\tilde{e}_{i}, \tilde{f}_{i}, i \in I$ given above.

(a) There is an embedding $\psi_{\mathbf{w}_{0}} B \hookrightarrow \mathbb{N}^{N}$, sending the graph $B(\infty)$ isomorphicaly onto the subgraph of $\mathcal{N}$ generated out of $\boldsymbol{u}_{\infty}:=(0,0, \ldots 0)$ by applying the operators $\tilde{e}_{i}, i \in I$.

(b) The image of $B(\infty)$ consists of those $\boldsymbol{a}=\left(a_{1}, a_{2}, \ldots, a_{N}\right) \in \mathbb{N}^{N}$ that verify

$$
\forall k=1 \ldots N: \quad \tilde{f}_{i_{k}}\left(\tilde{e}_{i_{k-1}}^{a_{k-1}} \tilde{e}_{i_{k-2}}^{a_{k-2}} \ldots \tilde{e}_{i_{1}}^{a_{1}} \boldsymbol{u}_{\infty}\right)=0 .
$$

Elements $\mathbf{a}:=\left(a_{1}, a_{2}, \ldots a_{N}\right)$ in $\left.\mathbf{b}\right)$ above are called string parameters [3]. The parametrization of set $B$ of vertices $B(\infty)$ obtained through Theorem 2.2 is Kashiwara's parametrization with respect to $\mathbf{w}_{0}$. Recall its indexing set $\operatorname{Im} \psi_{\mathbf{w}_{0}}$ is denoted by $\mathcal{S}_{\mathbf{w}_{0}}$.

REMARKS.

(i) Kashiwara works with the negative part $U_{q}\left(\mathfrak{n}^{-}\right)$of $U_{q}(\mathfrak{g})$. The above definition is the transfer of his construction to $U_{q}\left(\mathfrak{n}^{+}\right)$, which amounts to exchanging the roles of $\tilde{e}_{i}$ and $\tilde{f}_{i}$ at the level of $B(\infty)$.

(ii) The definition of Kashiwara's embedding imposes a reversal of order in the definition of a string, namely $\left(a_{1}, a_{2}, \ldots a_{N}\right)$ in our convention, corresponds to $\left(a_{N}, a_{N-1}, \ldots a_{1}\right)$ in [3].

THEOREM $2.3([\mathbf{1 4}, \mathbf{1 0}, \mathbf{4}])$. The set $\mathcal{S}_{\mathbf{w}_{0}}$ is the set of integer points of a polyhedral cone $\mathcal{C}_{\mathbf{w}_{0}}$, that is, there exists a finite set of vectors $K_{\mathbf{w}_{0}} \subset \mathbb{Z}^{N}$ such that

$$
\mathcal{S}_{\mathbf{w}_{0}}=\left\{\boldsymbol{a} \in \mathbb{N}^{N} \mid \forall \boldsymbol{k} \in K_{\mathbf{w}_{0}}, \boldsymbol{a} \cdot \boldsymbol{k} \geq 0\right\} .
$$

ExAmple. Consider type $A_{2}$, and $\mathbf{w}_{0}=s_{1} s_{2} s_{1}$. It is easy to compute the image of Kashiwara's embedding directly out of its definition above. One obtains the well-known result

$$
\mathcal{C}_{\mathbf{w}_{0}}=\left\{\left(a_{1}, a_{2}, a_{3}\right) \mid 0 \leq a_{1} \leq a_{2}, 0 \leq a_{3}\right\} .
$$

One may choose as a defining set for $\mathcal{C}_{\mathbf{w}_{0}}$, the set $K_{\mathbf{w}_{0}}=\{(1,0,0),(-1,1,0),(0,0,1)\}$, which is equal to both $K_{\mathbf{w}_{0}}^{G P}$ and $K_{\mathbf{w}_{0}}^{B Z}$.

MAIN THEOREM 2.4. Let $\mathbf{w}_{0}$ be a reduced expression adapted to a quiver of type $A_{n}$, and $K_{\mathbf{w}_{0}}^{G P}$ the set given by Gleizer and Postnikov [10, Section 5]. Then

$$
K_{\mathbf{w}_{0}}^{G P}=L_{\mathbf{w}_{0}} .
$$

Let us observe that in the Lusztig parametrization, the parameter set is the set of integer points of the cone $\left(\mathbb{R}^{+}\right)^{N}$, consisting of vectors with positive coordinates. This cone may be seen as being defined by the natural basis $E=\left\{\mathbf{e}_{1}, \mathbf{e}_{2} \ldots \mathbf{e}_{N}\right\}$ of $\mathbb{R}^{N}$. It is easy to see, by the definition of Kashiwara's embedding, that the set $E$ is the set of of moves of crystal operators $\tilde{e}_{i}, i \in I$ in Kashiwara's parametrization according to $\mathbf{w}_{0}$. We have therefore a full symmetry between Lusztig's and Kashiwara's parametrizations, the set of vectors defining the parameters set in one picture being equal to the set of moves occurring in crystal operators action in the other. 
CONJECTURE. Let $Q$ be a quiver of type ADE satisfying Reineke's condition $(L)$. Let $\mathbf{w}_{0}$ be adapted to it. Then $L_{\mathbf{w}_{0}}$ is a defining set for $\mathcal{C}_{\mathbf{w}_{0}}$.

We state this conjecture on the basis of some computer testing with GAP4 [8], using the set $K_{\mathbf{w}_{0}}^{B Z}$. We give in Section 7 a detailed $D_{4}$ example. The conjecture might be valid in a larger scope, even beyond reduced expressions adapted to quivers. However, one faces a breakdown of many nice properties, enjoyed by reduced expressions adapted to quivers verifying condition $(L)$.

3. Auslander-Reiten quivers and Lusztig's moves. Fix $Q$ a quiver of type $A D E$ satisfying condition $(L), \mathbf{w}_{0}$ adapted to it and $\beta_{1}, \beta_{2}, \ldots, \beta_{N}$ the reflection ordering it defines. The category $\bmod \mathbb{C} Q$ is equivalent to that of finite-dimensional representations of $Q$. A module $M$ in $\bmod \mathbb{C} Q$ may be seen as a family $\left(V_{i}\right)_{i \in I}$ of finite-dimensional $\mathbb{C}$-vector spaces, together with linear mappings $f_{i, j}: V_{i} \longrightarrow V_{j}$ corresponding to the arrows $i \longrightarrow j$ of $Q$. The dimension vector of $M$ is the element of $Q^{+}$given by $\mathbf{d}_{M}:=\sum_{i=1}^{n}\left(\operatorname{dim} V_{i}\right) \alpha_{i}$. A simple object $S_{i}$ has a dimension vector equal to $\alpha_{i}$.

Let Ind $Q$ denote the set of isomorphism classes of indecomposable objects of $\bmod \mathbb{C} Q$. The theorem of Gabriel states that for each $\beta \in \Phi^{+}$there is a unique class $[M] \in$ Ind $Q$ with $\mathbf{d}_{M}=\beta$, and that all indecomposable objects of $\bmod \mathbb{C} Q$ are obtained this way. There is, therefore, a one-to-one correspondence between Ind $Q$ and $\Phi^{+}$, and we shall denote by $[\beta]$ the class $[M]$ in Ind $Q$ whose dimension vector is $\beta$.

The Auslander-Reiten quiver $\Gamma_{Q}([\mathbf{1}$, Chapter VII $]$ and $[\mathbf{9}$, Section 6]) has a set of vertices Ind $Q$, and its arrows are irreducible morphisms between objects of Ind $Q$. It has a rigid mesh structure, as given in [9, Figure 13, p. 49]. The quiver $\Gamma_{Q}$ is endowed with the translation $\tau[\mathbf{1}$, p. 225], which sends non-projective modules of Ind $Q$ onto non-injective modules of Ind $Q$. The translation $\tau$ stratifies $\Gamma_{Q}$ into levels. The $i$ th level is the orbit under $\tau$ of the injective envelope of $S_{i^{*}}$, where $*$ denotes the Dynkin diagram automorphism induced by $w_{0}$. This level ends in the projective cover of $S_{i}$.

There is a natural order on the vertices of $\Gamma_{Q}$, given by $\left[\beta_{1}\right] \leq_{Q}\left[\beta_{2}\right]$ if and only if there is a path from $\left[\beta_{1}\right]$ to $\left[\beta_{2}\right]$ in $\Gamma_{Q}$. This induces a partial order $\preccurlyeq Q$ on $\Phi^{+}$by putting $\beta_{1} \preccurlyeq Q \beta_{2}$ whenever $\left[\beta_{1}\right] \leq_{Q}\left[\beta_{2}\right]$. The reflection ordering $\preccurlyeq_{\mathbf{w}_{0}}$ is then a linear refinement of $\preccurlyeq Q$.

The path algebra $\mathbb{C} Q$ is an hereditary algebra. The Euler-Poincaré characteristic $\left\langle M_{1}, M_{2}\right\rangle:=\operatorname{dim} \operatorname{Hom}\left(M_{1}, M_{2}\right)-\operatorname{dim} \operatorname{Ext}^{1}\left(M_{1}, M_{2}\right)$ depends only on the dimension vectors $\mathbf{d}_{M_{1}}$ and $\mathbf{d}_{M_{2}}$ of $M_{1}$ and $M_{2}$, respectively. One has $\left\langle\left[\beta_{1}\right],\left[\beta_{2}\right]\right\rangle=\left(\beta_{1}, \beta_{2}\right)_{R}$, where $(,)_{R}$ is the Ringel form upon the Euclidean space $\mathbb{R} \Phi$. The matrix $R=\left(r_{i, j}\right)$ of this form, on the basis of simple roots, is given by

$$
r_{i, j}:=\left(\alpha_{i}, \alpha_{j}\right)_{R}=\left\{\begin{array}{cl}
1 & \text { if } i=j \\
-1 & \text { if } i \longrightarrow j \text { is in Q } . \\
0 & \text { otherwise }
\end{array}\right.
$$

THEOREM 3.1 ([21]).

(i) Suppose $\beta_{1} \preccurlyeq Q \beta_{2}$, then $\operatorname{dim} \operatorname{Ext}^{1}\left(\left[\beta_{1}\right],\left[\beta_{2}\right]\right)=0$ and therefore $\operatorname{dim} \operatorname{Hom}\left(\left[\beta_{1}\right],\left[\beta_{2}\right]\right)=\left(\beta_{1}, \beta_{2}\right)_{R}$.

(ii) Suppose $\beta_{1} \succcurlyeq_{Q} \beta_{2}$, then $\operatorname{dim} \operatorname{Hom}\left(\left[\beta_{1}\right],\left[\beta_{2}\right]\right)=0$ and therefore $\operatorname{dim} \operatorname{Ext}^{1}\left(\left[\beta_{1}\right],\left[\beta_{2}\right]\right)=-\left(\beta_{1}, \beta_{2}\right)_{R}$. 
Theorem 3.1 reduces the testing of condition $(L)$ for a quiver $Q$, to computations in terms of $\Phi^{+}$.

We refer to Section 2 of [18] for a concise description of the Hall algebra construction $[19,20]$ of $U_{q}\left(\mathfrak{n}^{+}\right)$and its link to PBW bases. The $\mathbb{C}(q)$ vector space with formal base vectors $\mathbf{u}_{[M]}$ indexed by isomorphism classes of $\bmod \mathbb{C} Q$ may be endowed with a product linked to the module structure. This defines the Hall algebra $\mathcal{H}(Q)$. Ringel's main theorem [20] states that sending the generators $e_{i}$ to $\mathbf{u}_{\left[S_{i}\right]}$ establishes an isomorphism $\eta_{Q}: U_{q}\left(\mathfrak{n}^{+}\right) \stackrel{\sim}{\longrightarrow} \mathcal{H}(Q)$.

Let $[M]=\bigoplus_{j=1}^{N}\left[\beta_{j}\right]^{\oplus t_{j}}$ be an isoclass with multiplicities of indecomposables given by $\mathbf{t}_{M}:=\left(t_{1}, t_{2}, \ldots t_{N}\right)$. The PBW monomial $\mathrm{p}_{\mathbf{w}_{0}}\left(\mathbf{t}_{M}\right)$ with $\mathbf{w}_{0}$ adapted to $Q$ is recovered up to a multiplication by a well-defined power of $q$ as the inverse image under $\eta_{Q}$ of $\mathbf{u}_{[M]}$. Crystal operators in the Lusztig parametrization of $\mathbf{w}_{0}$ may therefore be seen as acting upon isomorphism classes of $\bmod \mathbb{C} Q$. One has $\tilde{e}_{i}\left[M_{1}\right]=\left[M_{2}\right]$ if and only if $\tilde{e}_{i} \mathrm{p}_{\mathbf{w}_{0}}\left(\mathbf{t}_{M_{1}}\right)=\mathrm{p}_{\mathbf{w}_{0}}\left(\mathbf{t}_{M_{2}}\right) \bmod q^{-1} \mathcal{L}$.

Let us fix $i \in I$. The description of the action of $\tilde{e}_{i}$ is given in terms of the set $[18$, p. 711]:

$$
P_{i}(Q):=\left\{[X] \in \operatorname{Ind} Q \mid \operatorname{dim} \operatorname{Hom}\left(X, S_{i}\right)>0\right\}
$$

The set $P_{i}(Q)$ has a poset structure $[X] \leq[Y]$ whenever there is a path from $[X]$ to $[Y]$ inside $P_{i}(Q)$. It is the same as the order induced by $\leq_{Q}$ [18, Proposition 4.3]. Recall that an antichain $A$ of a $P_{i}(Q)$ is a set of mutually non-comparable elements. It defines the order ideal $J(A):=\left\{[X] \in P_{i}(Q) \mid \exists[Y] \in A,[X] \leq[Y]\right\}$. The correspondence $A \mapsto J(A)$ is one-to-one, and inclusion between order ideals induces a natural poset structure upon the set $\mathcal{A}_{i}(Q)$ of all antichains of $P_{i}(Q)$.

Given $A \in \mathcal{A}_{i}(Q)$, let $C_{A}$ be the set of minimal elements of $P_{i}(Q) \backslash J(A)$, and define

$$
\begin{aligned}
& {\left[V_{A}\right]:=\underset{[M] \in A}{\oplus}[M],} \\
& {\left[U_{A}\right]:=\underset{[M] \in C_{A}}{\bigoplus}[\tau M] .}
\end{aligned}
$$

The Lusztig move corresponding to $A$ is then $\mathbf{l}_{A}:=\mathbf{t}_{V_{A}}-\mathbf{t}_{U_{A}}$.

Each $A \in \mathcal{A}_{i}(Q)$ also defines a function $F_{A}: \mathbb{N}^{N} \longrightarrow \mathbb{Z}$ given by

$$
F_{A}(\mathbf{t}):=\sum_{X \in J(A)} \Delta_{X}(\mathbf{t})
$$

where for $X=\left[\beta_{k}\right]$ with $\tau X=\left[\beta_{k^{\prime}}\right], \Delta_{X}(\mathbf{t})=t_{k}-t_{k^{\prime}}$ (with the convention that the second term is 0 if translation is not defined on $X$ ).

Theorem 3.2 ([18, Theorem 7.1]). Let $[M]$ be an isomorphism class of mod $\mathbb{C} Q$. Put $\zeta_{i}([M]):=\max _{A \in \mathcal{A}_{i}(Q)} F_{A}\left(\boldsymbol{t}_{M}\right)$. Then the subset $\left\{A \mid F_{A}\left(\boldsymbol{t}_{M}\right)=\zeta_{i}([M])\right\}$ of $\mathcal{A}_{i}(Q)$ admits a unique maximal element $A_{\text {max }}$. There is an isoclass $[X]$ such that $[M]=[X] \oplus\left[U_{A_{\max }}\right]$, and the action of $\tilde{e}_{i}$ is then given by $\tilde{e}_{i}[M]=[X] \oplus\left[V_{A_{\max }}\right]$.

In terms of the Lusztig parameters, $\tilde{e}_{i} \mathbf{t}_{M}=\mathbf{t}_{M}+\mathbf{l}_{A_{\max }}$. 
Corollary 3.3. Consider the Lusztig parametrization corresponding to $\mathbf{w}_{0}$. The set of Lusztig moves of type $i$ is given by

$$
L_{\mathbf{w}_{0}}^{(i)}=\left\{\mathbf{l}_{A} \mid A \in \mathcal{A}_{i}(Q)\right\}
$$

Proof. In view of Theorem 3.2, given $A \in \mathcal{A}_{i}(Q)$, we need to exhibit a module on which $\tilde{e}_{i}$ acts according to the move defined by $A$. Such a module is $U_{A}$. One has $\Delta_{X}\left(\mathbf{t}_{U_{A}}\right) \geq 0$ for any $[X] \in J(A)$, where as $\Delta_{X}\left(\mathbf{t}_{U_{A}}\right)=-1$ for any $[X] \in C_{A}$. These two properties ensure that for $U_{A}, A_{\max }$ of Theorem 3.2 is $A$.

ExAmPle. Let $Q$ be the quiver ${ }^{1} \longleftarrow^{2} \cdot \longrightarrow^{3} \cdot{ }^{3}$ of type $A_{3}$ with adapted reduced expression

$\mathbf{w}_{0}=s_{1} s_{3} s_{2} s_{1} s_{3} s_{2}$. The Auslander-Reiten quiver $\Gamma_{Q}$ is

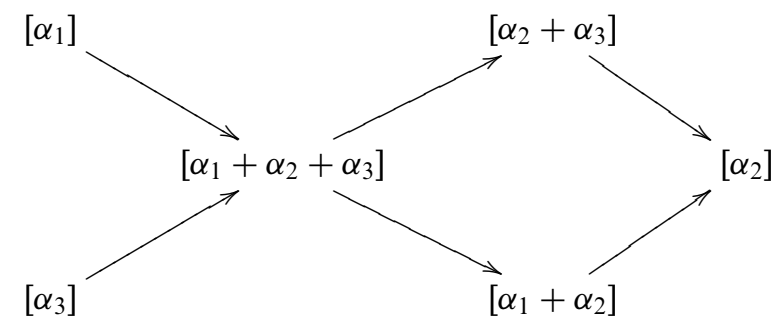

Let us study the action of $\tilde{e}_{2}$. Using $(,)_{R}$ and Theorem 3.1, one gets $P_{2}(Q)=\left\{\left[\alpha_{2}\right],\left[\alpha_{1}+\alpha_{2}\right],\left[\alpha_{2}+\alpha_{3}\right],\left[\alpha_{1}+\alpha_{2}+\alpha_{3}\right]\right\} . \mathcal{A}_{2}(Q)$ consists in five antichains:

\begin{tabular}{|l|c|c|c|} 
Antichain $A$ & $U_{A}$ & $V_{A}$ & $\mathbf{l}_{A}$ \\
\hline$A_{1}=\left\{\left[\alpha_{1}+\alpha_{2}+\alpha_{3}\right]\right\}$ & {$\left[\alpha_{1}\right] \oplus\left[\alpha_{3}\right]$} & {$\left[\alpha_{1}+\alpha_{2}+\alpha_{3}\right]$} & $(-1,-1,1,0,0,0)$ \\
$A_{2}=\left\{\left[\alpha_{2}+\alpha_{3}\right]\right\}$ & {$\left[\alpha_{3}\right]$} & {$\left[\alpha_{2}+\alpha_{3}\right]$} & $(0,-1,0,1,0,0)$ \\
$A_{3}=\left\{\left[\alpha_{1}+\alpha_{2}\right]\right\}$ & {$\left[\alpha_{1}\right]$} & {$\left[\alpha_{1}+\alpha_{2}\right]$} & $(-1,0,0,0,1,0)$ \\
$A_{4}=\left\{\left[\alpha_{1}+\alpha_{2}\right],\left[\alpha_{2}+\alpha_{3}\right]\right\}$ & {$\left[\alpha_{1}+\alpha_{2}+\alpha_{3}\right]$} & {$\left[\alpha_{1}+\alpha_{2}\right] \oplus\left[\alpha_{2}+\alpha_{3}\right]$} & $(0,0,-1,1,1,0)$ \\
$A_{5}=\left\{\left[\alpha_{2}\right]\right\}$ & 0 & {$\left[\alpha_{2}\right]$} & $(0,0,0,0,0,1)$ \\
\hline
\end{tabular}

The set $\mathcal{A}_{2}(Q)$ has the following poset structure:

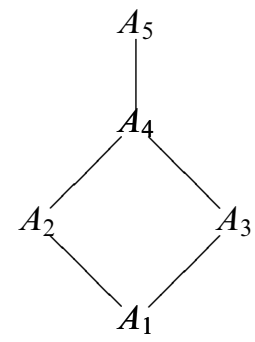

Considering the structure of $\Gamma_{Q}$ and the order ideals defined by members of $\mathcal{A}_{2}(Q)$, we get the $F$-functions data. 


\begin{tabular}{c|l|l} 
Antichain & $J(A)$ & $F_{A}$ \\
\hline$A_{1}$ & $\left\{\left[\alpha_{1}+\alpha_{2}+\alpha_{3}\right]\right\}$ & $t_{3}$ \\
$A_{2}$ & $\left\{\left[\alpha_{1}+\alpha_{2}+\alpha_{3}\right],\left[\alpha_{2}+\alpha_{3}\right]\right\}$ & $t_{3}+\left(t_{4}-t_{1}\right)$ \\
$A_{3}$ & $\left\{\left[\alpha_{1}+\alpha_{2}+\alpha_{3}\right],\left[\alpha_{1}+\alpha_{2}\right]\right\}$ & $t_{3}+\left(t_{5}-t_{2}\right)$ \\
$A_{4}$ & $\left\{\left[\alpha_{1}+\alpha_{2}+\alpha_{3}\right],\left[\alpha_{1}+\alpha_{2}\right],\left[\alpha_{2}+\alpha_{3}\right]\right\}$ & $t_{3}+\left(t_{4}-t_{1}\right)+\left(t_{5}-t_{2}\right)$ \\
$A_{5}$ & $P_{2}(Q)$ & $\left(t_{4}-t_{1}\right)+\left(t_{5}-t_{2}\right)+t_{6}$ \\
\hline
\end{tabular}

Take $\mathbf{t}_{M}=(3,2,1,1,2,0)$. One has $t_{3}=1, t_{4}-t_{2}=-2, t_{5}-t_{2}=0, t_{6}=0$. Replacing each antichain $A$ by the value of $F_{A}\left(\mathbf{t}_{M}\right)$ gives the following diagram:

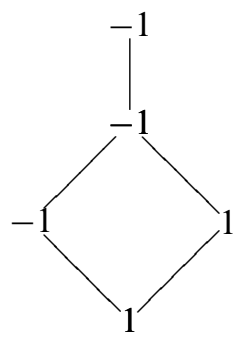

This pinpoints $A_{3}$ as $A_{\max }$ for $[M]$. Thus, $\tilde{e}_{2} \mathbf{t}_{M}=\mathbf{t}_{M}+\mathbf{l}_{A_{3}}=(2,2,1,1,3,0)$.

4. Wiring diagrams and string cones. We shall restrict ourselves from now on to $A_{n}$ type. The positive roots are $\beta=\alpha_{i}+\alpha_{i+1}+\cdots+\alpha_{j}, 1 \leq i \leq j \leq n$. They are in a one-to-one correspondence with pairs $(i, j), 1 \leq i<j \leq n+1, \beta$ above being sent to $(i, j+1)$. The fundamental representation $E\left(\omega_{1}\right)$ of $\mathfrak{s l}_{n+1}$ has weights given by $v_{j}=-\omega_{j-1}+\omega_{j}, j=1 \cdots n+1$ (with the convention that $\omega_{0}=\omega_{n+1}=0$ ). The weight $v_{j}$ may be seen as the weight of a one-box Young tableau $j$. The weights of fundamental representations $E\left(\omega_{k}\right)$ for $2 \leq k \leq n$ correspond to strictly increasing column tableaux of size $k$. The weight of a tableau is the sum of weights of its boxes. We shall therefore identify these weights with $k$-tuples $1<j_{1}<j_{2}<\cdots<j_{k} \leq n+1$. The action of $W$ on roots or on weights identifies with that of the symmetric group $\mathfrak{S}_{n+1}$ on the respective multi-indices that we considered.

The wiring diagram $\mathcal{W D}\left(\mathbf{w}_{0}\right)$ of a reduced expression $\mathbf{w}_{0}=s_{i_{1}} s_{i_{2}} \cdots s_{i_{N}}$ consists in encoding $\mathbf{w}_{0}$ as an arrangement of pseudo-lines $L_{1}, \ldots, L_{n+1}$ drawn inside a vertical strip of $\mathbb{R}^{2}$. The respective crossing points of these strands occur in levels according to the indices of $\mathbf{w}_{0}$. Figure 1 gives a self-explaining example of the procedure in type $A_{3}$.

Each strand $L_{i}$ crosses another strand $L_{j}$ once and only once. The order of the strands $L_{i}, i=1, \ldots n+1$ gets inverted while following $\mathcal{W D}\left(\mathbf{w}_{0}\right)$ from left to right. Sending the crossing $v_{i, j}(i<j)$ of lines $L_{i}$ and $L_{j}$ onto the couple $(i, j)$ establishes a one-to-one order preserving correspondence between these crossings enumerated from left to right and the reflection ordering $\preccurlyeq w_{w_{0}}$ of $\Phi^{+}$. If $\beta_{k}$ is the $k$ th root in that order, with $i_{k}=i$, then the $k$ th crossing of $\mathcal{W D}\left(\mathbf{w}_{0}\right)$, which we denote by $v_{\beta_{k}}$, is on level $i$.

Let $G^{\circ}\left(\mathbf{w}_{0}\right)$ be the non-oriented graph obtained from $\mathcal{W D}\left(\mathbf{w}_{0}\right)$, whose vertices are the crossing points of pseudolines, and whose edges are given by pseudoline segments linking two crossing points. Likewise, let us denote by $G\left(\mathbf{w}_{0}\right)$ the non-oriented graph obtained in a similar way by considering the vertices of $G^{\circ}\left(\mathbf{w}_{0}\right)$ as well as the vertices $l_{1}, \ldots, l_{n+1}, r_{1}, \ldots r_{n+1}$ on the border of $\mathcal{W D}\left(\mathbf{w}_{0}\right)$. 


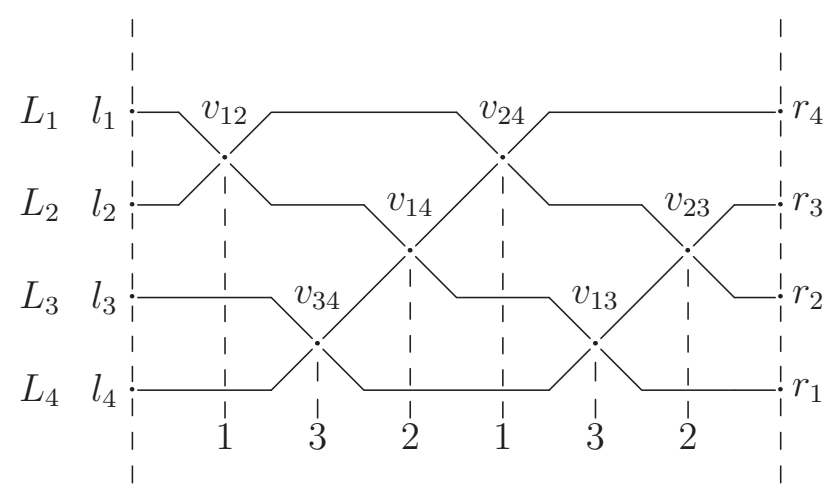

Figure 1. Wiring diagram of $\mathbf{w}_{0}=s_{1} s_{3} s_{2} s_{1} s_{3} s_{2}$ of type $A_{3}$.

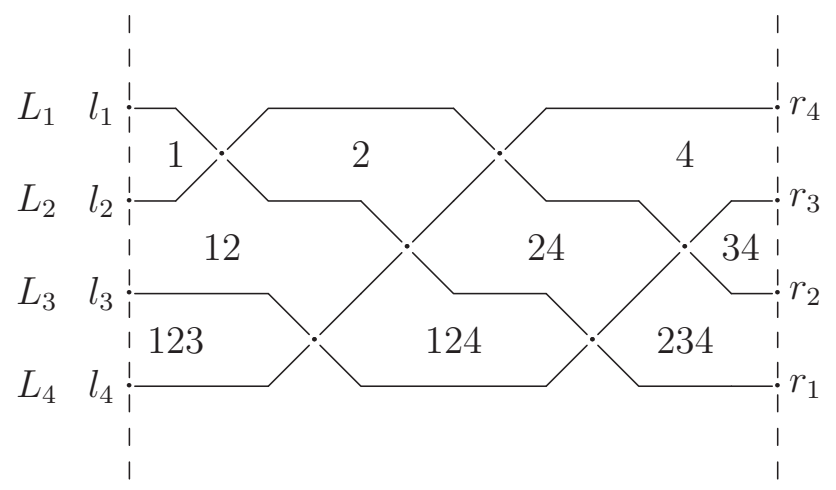

Figure 2. Chamber system of $\mathcal{W D}\left(\mathbf{w}_{0}\right)$ for $\mathbf{w}_{0}=s_{1} s_{3} s_{2} s_{1} s_{3} s_{2}$ of type $A_{3}$.

Two vertices $v_{\beta_{k}}$ and $v_{\beta_{k^{\prime}}}$ with $k<k^{\prime}, i_{k}=i$ and $i_{k^{\prime}}=j$ are adjacent in $G^{\circ}\left(\mathbf{w}_{0}\right)$ if one of the two cases occur. The adjacency is diagonal if one has $|i-j|=1$ and $i_{l} \neq i, i_{l} \neq j$ for any $l$ satisfying $k<l<k^{\prime}$. The adjacency is horizontal when $i=j$ and either $i_{l}<i-1$ for all $k<l<k^{\prime}$ or $i_{l}>i+1$ for all $k<l<k^{\prime}$. We see in Figure 1 that $v_{12}$ and $v_{14}$ are diagonally adjacent, whereas $v_{12}$ and $v_{24}$ are horizontally adjacent.

The wiring diagram $\mathcal{W D}\left(\mathbf{w}_{0}\right)$ defines a set of bounded chambers. Such a chamber may be indexed by a set of indices of pseudolines passing above it. This set may be seen as indices of a column Young tableau. One gets a one-to-one correspondence between these chambers and a set of weights inside $\bigcup_{k=1}^{n} W \omega_{k}$.

A vertex $v_{\beta}$ of $G^{\circ}\left(\mathbf{w}_{0}\right)$ may be assigned either the weight $\lambda^{-}\left(v_{\beta}\right)$ of the chamber left to it or the weight $\lambda^{+}\left(v_{\beta}\right)$ of the chamber right to it. It is well known that if $\beta=s_{i_{1}} s_{i_{2}} \cdots s_{i_{k-1}}\left(\alpha_{i_{k}}\right)$, then

$$
\lambda^{-}(\beta)=s_{i_{1}} s_{i_{2}} \cdots s_{i_{k-1}}\left(\omega_{i_{k}}\right), \quad \lambda^{+}(\beta)=s_{i_{1}} s_{i_{2}} \cdots s_{i_{k-1}} s_{i_{k}}\left(\omega_{i_{k}}\right)
$$

Figure 2 gives the example of the chamber system for $\mathbf{w}_{0}=s_{1} s_{3} s_{2} s_{1} s_{3} s_{2}$ of type $A_{3}$. The vertex $v_{14}$ is between the chamber labelled 12 to the left, and that labelled 24 to the right. One has $\lambda^{-}\left(v_{14}\right)=v_{1}+v_{2}, \lambda^{+}\left(v_{14}\right)=v_{2}+v_{4}$.

Gleizer-Postnikov [10, Section 5] obtain a system of defining inequalities for the string cone $\mathcal{C}_{\mathbf{w}_{0}}$ by transforming for every $i \in I$ the graph $G\left(\mathbf{w}_{0}\right)$ into an oriented graph 


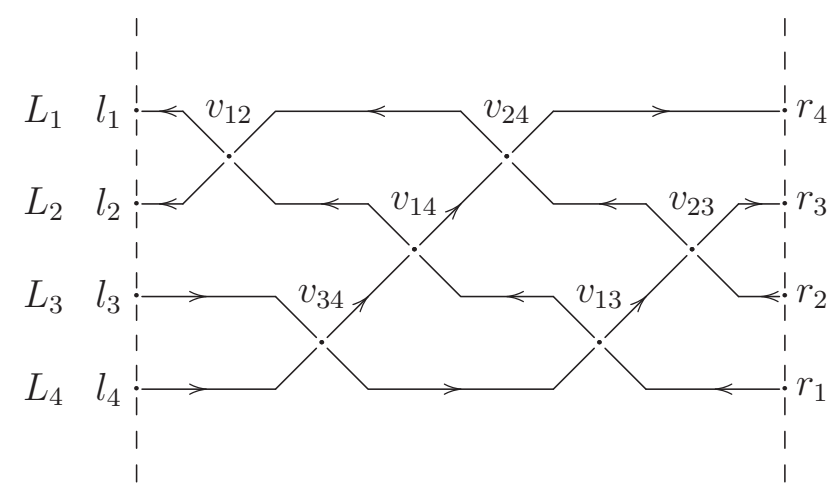

Figure 3. $G\left(\mathbf{w}_{0}, 2\right), \mathbf{w}_{0}=s_{1} s_{3} s_{2} s_{1} s_{3} s_{2}$, type $A_{3}$.

$G\left(\mathbf{w}_{0}, i\right)$. The pseudolines $L_{1}, L_{2}, \ldots L_{i}$ are oriented backwards, and the pseudolines $L_{i+1}, \ldots L_{n+1}$ are oriented forwards. The resulting graph is acyclic.

Let $\pi$ be a path inside $G\left(\mathbf{w}_{0}, i\right)$. We shall qualify the two configurations below as forbidden crossings:

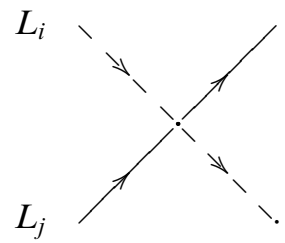

(path $\pi$ in plain line)

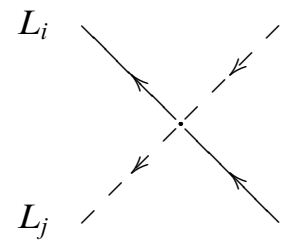

$L_{j}$ 
$L_{4}$ to $L_{2}$ (hence a positive contribution). The vertices $v_{13}, v_{14}, v_{24}$ occur respectively in positions 5, 3 and 4, while we follow $\mathcal{W D}\left(s_{1} s_{3} s_{2} s_{1} s_{3} s_{2}\right)$ from left to right. We get $\mathbf{k}_{\pi}=(0,0,-1,1,1,0)$.

As $G\left(\mathbf{w}_{0}, i\right)$ is acyclic, there are only finitely many different GP paths of type $i$.

THEOREM 4.1 ([10, Corollary 5.8]). Let $K_{\mathbf{w}_{0}}^{G P}$ be the set of all vectors $\boldsymbol{k}_{\pi}$, where $\pi$ varies over all possible GP-paths of all possible types $i \in I$. Then $K_{\mathbf{w}_{0}}^{G P}$ defines the string cone $\mathcal{C}_{\mathrm{w}_{0}}$. One has

$$
\mathcal{S}_{\mathbf{w}_{0}}=\left\{\boldsymbol{t} \in \mathbb{N}^{N} \mid \forall \boldsymbol{k}_{\pi} \in K_{\mathbf{w}_{0}}^{G P}, \quad \boldsymbol{k}_{\pi} \cdot \boldsymbol{t} \geq 0\right\} .
$$

Fix $i \in I$, and consider $G\left(\mathbf{w}_{0}, i\right)$. Let us denote by $\delta_{i}^{>}$the segment of $L_{i+1}$ starting from the left border on $l_{i+1}$ up to its intersection $v_{\alpha_{i}}$ with $L_{i}$. In a similar way, we denote by $\delta_{i}^{<}$the segment of $L_{i}$ starting from $v_{\alpha_{i}}$ and going back to $l_{i}$ on the left border following $L_{i}$. The concatenation $\delta_{i}:=\delta_{i}^{>} * \delta_{i}^{<}$is then a path starting from $l_{i+1}$ and ending in $l_{i}$. We shall call it the limiting path of type $i$.

Observe $\delta_{i}$ serves as a boundary of a set of chambers $\mathcal{Z}_{i}\left(\mathbf{w}_{0}\right)$. These chambers are those that lie below $L_{i}$, above $L_{i+1}$ and left of $v_{\alpha_{i}}$. Let us denote by $Z_{i}\left(\mathbf{w}_{0}\right)$ the set of vertices of $G^{\circ}\left(\mathbf{w}_{0}\right)$, which are the rightmost vertices of chambers of $\mathcal{Z}_{i}\left(\mathbf{w}_{0}\right)$.

Lemma 4.2. Consider $v$ a vertex of $\delta_{i}$ other than $l_{i}, l_{i+1}, v_{\alpha_{i}}$. Suppose this vertex is a crossing of $\delta_{i}$ with a line $L_{k}(k \neq i, i+1)$. We have then the following two cases:

(i) If $v \in \delta_{i}^{>}$, then $L_{k}$ crosses $\delta_{i}$ going inside $\mathcal{Z}_{i}\left(\mathbf{w}_{0}\right)$.

(ii) If $v \in \delta_{i}^{<}$, then $L_{k}$ crosses $\delta_{i}$ going outside $\mathcal{Z}_{i}\left(\mathbf{w}_{0}\right)$.

\section{Proof of Lemma 4.2.}

(i) We have to eliminate the possibility of $L_{k}$ going out of $\mathcal{Z}_{i}\left(\mathbf{w}_{0}\right)$ while crossing $\delta_{i}^{>}$. By definition, $\mathcal{Z}_{i}\left(\mathbf{w}_{0}\right)$ lies above $\delta_{i}^{>}$, hence $L_{k}$ crosses $\delta_{i}^{>}$going downwards. Depending on the orientation of $L_{k}$, we obtain the following two cases:

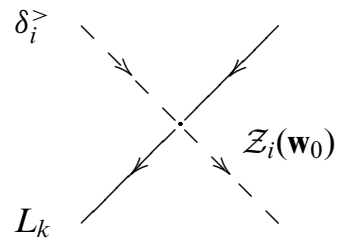

(a) $k<i$

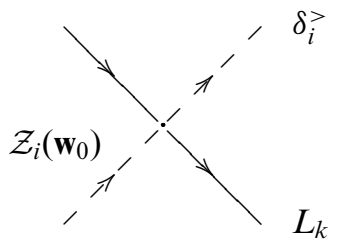

(b) $k>i+1$

In case (a), $L_{k}$ would have to recross $L_{i+1}$ in order to return to $l_{k}$, which lies above $l_{i+1}$ on the left border. In case (b), $l_{k}$ lies below $l_{i+1}$ on the left border, so $L_{k}$ would have to cross $L_{i+1}$ for first time in order to reach the crossing point in (b) from above. Both cases are impossible since they would imply at least two crossings of $L_{k}$ and $L_{i+1}$ in $\mathcal{W D}\left(\mathbf{w}_{0}\right)$.

Statement (ii) is proved by symmetric arguments.

COROLlary 4.3. $\delta_{i}$ is a GP-path.

Proof. Clearly, $v_{\alpha_{i}}$ is not a forbidden crossing. The other vertices of $\delta_{i}$ belong to the cases detailed in Lemma 4.2, none of them being forbidden. 
Proposition 4.4. Let $\pi$ be a GP-path of type $i$. Then $\pi$ stays inside $\mathcal{Z}_{i}\left(\mathbf{w}_{0}\right)$.

Proof.

The path $\pi$ starts at $l_{i+1}$ and ends at $l_{i}$, which are inside $\mathcal{Z}_{i}\left(\mathbf{w}_{0}\right)$. Suppose it exits $\mathcal{Z}_{i}\left(\mathbf{w}_{0}\right)$ at some vertex $v_{1} \in \delta_{i}$. By Lemma 4.2 , one must have $v_{1} \in \delta_{i}^{<}$. The same lemma shows $\pi$ must return inside $\mathcal{Z}_{i}\left(\mathbf{w}_{0}\right)$ through a crossing point $v_{2} \in \delta_{i}^{>}$. Now the segment of $\delta_{i}$ between $v_{1}$ and $v_{2}$ goes from $v_{2}$ to $v_{1}$, so we can create a cycle. This is in contradiction with the fact that $G\left(\mathbf{w}_{0}, i\right)$ is acyclic.

5. Hammocks. We continue to restrict ourselves to $A_{n}$ case, with $Q$ and $\mathbf{w}_{0}$ fixed. Let us denote by $\left[\beta: \alpha_{i}\right]$ the coefficient of $\alpha_{i}$ in the expression of $\beta$. The hammock of type $i, i \in I[6]$ is $H_{i}(Q):=\left\{[\beta] \mid\left[\beta: \alpha_{i}\right]>0\right\}$. The set $P_{i}(Q)$ is a subset of $H_{i}(Q)$, and we shall see that the combinatorics of Lusztig moves of type $i$ is obtained from that of $H_{i}(Q)$. The structure of $H_{i}(Q)$ itself is very simple, and is deduced from the Coxeter element $c$ attached to $Q$.

Recall [5] that one may renumber the vertices of $Q$ by a permutation $i_{1}, i_{2}, \ldots, i_{n}$ of $I$ such that for every arrow $j \longrightarrow k$ of $Q$ one has $i_{j}<i_{k}$. The Coxeter element is then given by $c=s_{i_{1}} s_{i_{2}} \cdots s_{i_{n}}$. The action of $c$ on $\Phi^{+}$is the mirror image of the action of the translation $\tau$ upon $\Gamma_{Q}$ : if $N=\tau M$ then $\mathbf{d}_{N}=c \mathbf{d}_{M}$.

In type $A_{n}$, as $W \cong \mathfrak{S}_{n+1}, c$ is an $(n+1)$-cycle. Its expression may be constructed out of $Q$ by the following algorithm [22, Lemma 4.2]:

- Start with just the element $n+1$.

- Proceed in decreasing order $i=n, n-1, \ldots, 2$ :

- If inside $Q$, one has $\stackrel{i-1}{\cdot} \longleftarrow^{i}$, add $i$ to the right of the indices already written.

- If inside $Q$, one has $\stackrel{i-1}{\longrightarrow} \stackrel{i}{\longrightarrow}$, add $i$ to the left of the indices already written.

- Finish by adding 1 to the left of the $n$ indices already written.

The sequence of indices $j_{1} j_{2} \cdots j_{n+1}$ thus obtained is a $(n+1)$-cycle expression of $c$. The consequence of this specific algorithm is that the $(n+1)$-cycle expressions of $c$ verify a special 'segment' property.

Lemma 5.1 ([22, Proposition 4.3]).

For every $i \in\{1, \ldots, n\}$ there is a cycle expression $c=\left(j_{1}, \ldots, j_{i}, j_{i+1} \cdots j_{n+1}\right)$, where

- $j_{1}, j_{2}, \ldots j_{i}$ is a permutation of $1, \ldots i$.

- $j_{i+1}, j_{i+2}, \ldots j_{n+1}$ is a permutation of $i+1, i+2, \ldots n+1$.

We shall refer to this cycle expression as the $i$-segmented expression of $c$, and denote it by $\left(j_{1}, \ldots j_{i} \mid j_{i+1}, \ldots, j_{n+1}\right)$.

ExAMPLE. Consider the quiver $Q: \longleftarrow^{2} \cdot \longleftarrow^{2} \cdot{ }^{3} \longleftarrow^{4} \cdot$ of type $A_{4}$. One has $c=$ $s_{2} s_{1} s_{4} s_{3}$. The algorithm above gives

$$
5 \stackrel{\text { right }}{\longrightarrow} 54 \stackrel{\text { left }}{\longrightarrow} 354 \stackrel{\text { right }}{\longrightarrow} 3542 \stackrel{\text { left }}{\longrightarrow} 13542
$$

One may verify that the obtained five-cycle expression (13542) agrees with $c$. The $i$-segmented expressions of $c$ are then respectively

$$
\begin{array}{lll}
i=1:(1 \mid 3542) & i=3:(213 \mid 54) \\
i=2:(21 \mid 354) & i=4:(4213 \mid 5)
\end{array}
$$




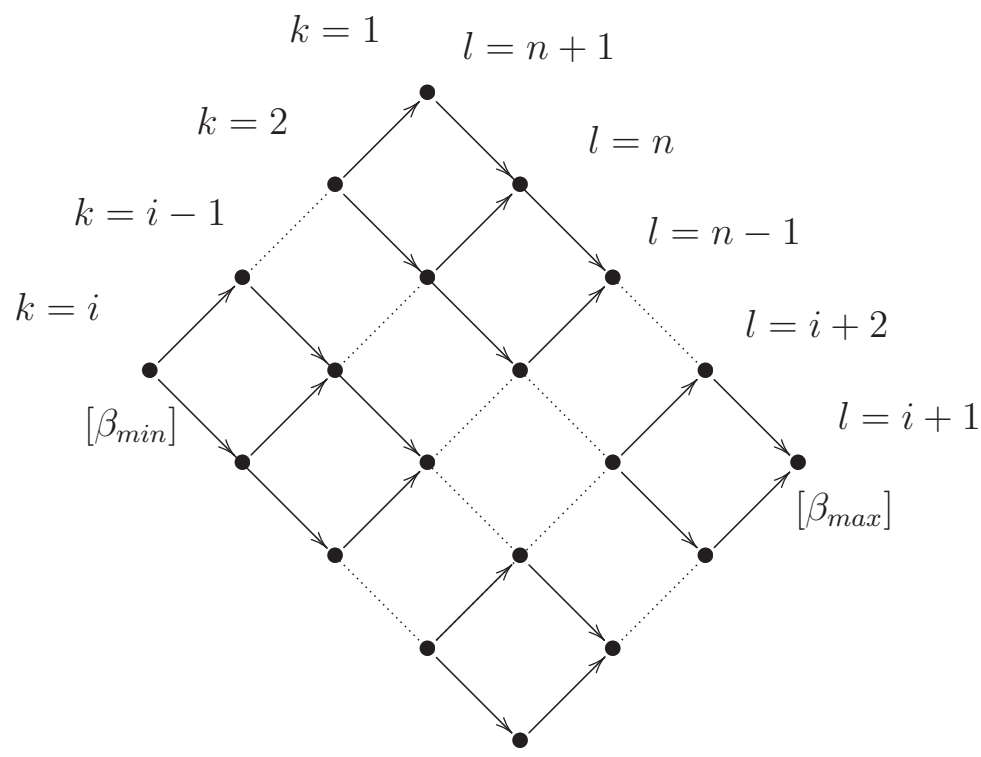

Figure 4. Hammock $H_{i}(Q)$ of type $A_{n}$.

The positions of $i, i+1$ in the $i$-segmented expression of $c$ depend on the neighbourhood of $i$ in $Q$ :

$$
\begin{array}{ll}
\stackrel{i-1}{\cdot} \longrightarrow \stackrel{i}{\longrightarrow} \text { in } \mathrm{Q}: j_{i}=i & \stackrel{i-1}{\cdot} \longleftarrow^{i} \cdot \text { in } \mathrm{Q}: j_{1}=i \\
\stackrel{i}{\longrightarrow} \stackrel{i+1}{\cdot} \text { in } \mathrm{Q}: j_{i+1}=i+1 \stackrel{i}{\cdot} \longleftarrow^{i+1} \cdot \text { in } \mathrm{Q}: j_{n+1}=i+1
\end{array}
$$

In the above example, the neighbourhood of vertex 2 in $Q$ is $\cdot \longleftarrow^{\longleftarrow^{\prime}} \cdot{ }^{\longrightarrow}{ }^{3} \cdot$ so that 2 appears in the first position of segment 21, and 3 appears in the first position of segment 354 .

Given an integer $m \geq 1$, we shall denote by $[m]$ the set $\{1,2, \ldots m\}$.

Proposition 5.2. Let $Q$ be a quiver of type $A_{n}$.

(a) The set $H_{i}(Q)$ for $i \in I$, seen as a subgraph of $\Gamma_{Q}$, has a structure isomorphic to $[i] \times[n+1-i]$ as given in Figure 4 , with $\left[\beta_{\min }\right]$ the isoclass of the projective cover of the simple module $S_{i}$ and $\left[\beta_{\max }\right]$ the isoclass of the injective envelope of $S_{i}$.

(b) If $\left(j_{1} j_{2} \cdots j_{i} \mid j_{i+1} \cdots j_{n+1}\right)$ is the $i$-segmented writing of $c$, then the vertex at position $(k, l)$ in the Figure 4 is $\left[\alpha_{j_{k}}+\alpha_{j_{k}+1}+\cdots \alpha_{j_{l}-1}\right]$.

The proposition consists in a computation well known to specialists. The reader may consult [9, Section 6.5, pp. 52-54] for technical details. Our Figure 4 corresponds to the first scheme in [9, Figure 15]. We provide here some guidelines for non-specialists.

The set $H_{i}(Q)$ is particularly simple to compute in the case of the ' $i$-regular' quiver $\stackrel{1}{\longrightarrow} \cdot \longrightarrow \ldots \longrightarrow \cdot \stackrel{i}{\longrightarrow} \ldots \longleftarrow^{n-1} \cdot \longleftarrow^{n} \cdot$ admitting $i$ as its unique sink. The modules at positions $(1, n+1),(2, n+1), \ldots,(i, n+1),(i, n), \ldots,(i, i+1)$ in Figure 4 are the respective projective covers of the simple modules $S_{1}, \ldots, S_{n}$. Their dimension vectors are directly obtained from $Q$.

The mesh structure of $\Gamma_{Q}$ [9] verifies additivity for dimension vectors. For each 'square' configuration of vertices $[X],\left[Z_{1}\right],\left[Z_{2}\right],[Y]$ respectively at positions 
$(k, l),(k-1, l),(k, l-1),(k-1, l-1)$ in Figure 4 , one has $\mathbf{d}_{X}+\mathbf{d}_{Y}=\mathbf{d}_{Z_{1}}+\mathbf{d}_{Z_{2}}$. Thus, the knowledge of the dimension vectors for the 'slice' of projective modules allows to compute the rest of $H_{i}(Q)$.

An arbitrary quiver $Q$ may be obtained from the $i$-regular one by a sequence of orientation changes transforming a source vertex $j$, with $j \neq i$, into a sink. The effect of this transformation on vertices of $\Gamma_{Q}$ other than $\left[\alpha_{j}\right]$ consists in applying the corresponding BGP-reflection functor $\Sigma_{j}$ [5]. This is the case of the vertices of $H_{i}(Q)$, which never contain $\left[\alpha_{j}\right]$. In terms of dimension vectors, $\mathbf{d}_{\Sigma_{j} X}=s_{j} \mathbf{d}_{X}$. One checks this change is coherent with the change in the Coxeter element $c$ due to the change of orientation.

One may verify by direct computation that $P_{i}(Q)$ is the order ideal defined by $\left[\alpha_{i}\right]$ inside $H_{i}(Q)$. The possible cases, according to the neighbourhood of $i$ inside $Q$, are:
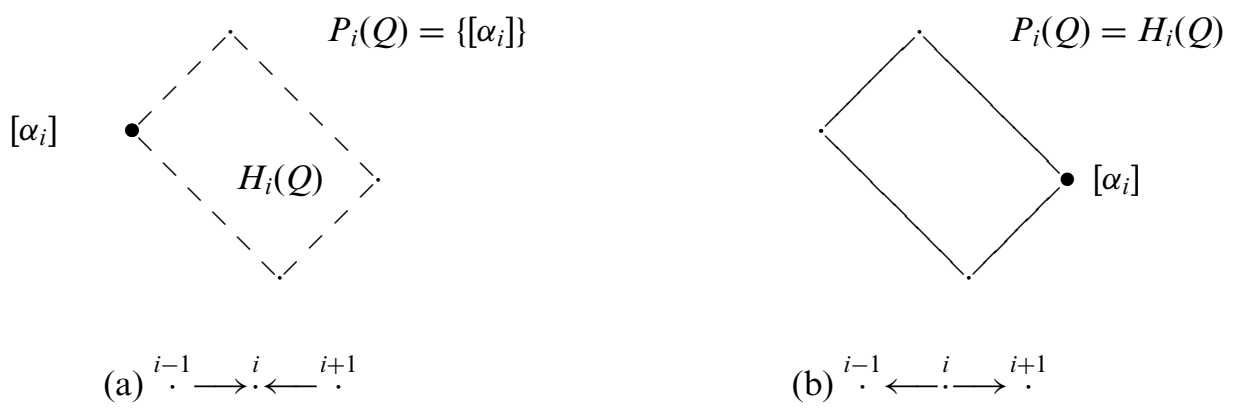

(b) $\stackrel{i-1}{\cdot} \longleftarrow \cdot i \cdot \longrightarrow^{i+1} \cdot$
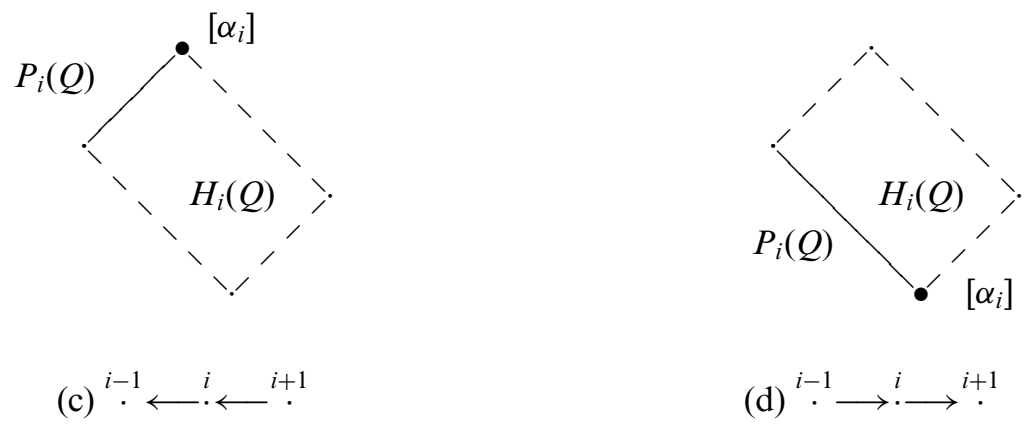

The correspondence $\Psi: \Gamma_{Q} \longrightarrow G^{\circ}\left(\mathbf{w}_{0}\right),[\beta] \mapsto v_{\beta}$ will allow us to transfer the above combinatorial results to the setting of $\mathcal{W D}\left(\mathbf{w}_{0}\right)$. In particular, the translation operation $\tau$ on $\Gamma_{Q}$ corresponds under $\Psi$ to an operation $\tau_{w d}$ on vertices of $G^{\circ}\left(\mathbf{w}_{0}\right)$. It is well known [2, Lemma 2.11] that for every $i \in I, \Psi$ establishes an order preserving one-to-one correspondence between the $i$ th translation level of $\Gamma_{Q}$ and crossings on the $i$ th level of $\mathcal{W D}\left(\mathbf{w}_{0}\right)$. Given $k$ between 1 and $N$, let $k^{-}$denote the maximal index $j$ such that $j<k$ and $s_{i_{j}}=s_{i_{k}}$ in $\mathbf{w}_{0}$ (if such an index exists). The isoclass $\left[\beta_{k}\right]$ is not projective exactly when $k^{-}$is defined. Then one has $\tau_{w d}\left(v_{\beta_{k}}\right)=v_{\beta_{k^{-}}}=v_{c \beta_{k}}$.

Let us define $H_{i}\left(\mathbf{w}_{0}\right):=\Psi\left(H_{i}(Q)\right)$.

Proposition 5.3. Fix $i \in I$ and consider $H_{i}(Q)$ and $H_{i}\left(\mathbf{w}_{0}\right)$ respectively as subgraphs of $\Gamma_{Q}$ and $G^{\circ}\left(\mathbf{w}_{0}\right)$. Then $\Psi$ restricted to $H_{i}(Q)$ establishes an isomorphism of non-oriented graphs between $H_{i}(Q)$ and $H_{i}\left(\mathbf{w}_{0}\right)$. 
Proof. Let $\left[\beta_{k}\right] \longrightarrow\left[\beta_{k^{\prime}}\right], k<k^{\prime}$ be an arrow of $H_{i}(Q)$. By [22, Proposition 1.2], the vertices $i_{k}$ and $i_{k^{\prime}}$ are linked in $D$, and there is no occurrence either of reflection $s_{i_{j}}=s_{i_{k}}$ or reflection $s_{i_{j}}=s_{i_{k^{\prime}}}$ in a position $j$ between $k+1$ and $k^{\prime}-1$. This means that $v_{\beta_{k}}$ and $v_{\beta_{k^{\prime}}}$ are diagonally adjacent inside $H_{i}\left(\mathbf{w}_{0}\right)$.

Suppose now $v_{\beta_{k}}$ and $v_{\beta_{k^{\prime}}}, k<k^{\prime}$, are adjacent vertices of $H_{i}\left(\mathbf{w}_{0}\right)$. A reduced expression $\mathbf{w}_{0}$ adapted to a quiver is alternating [22, Lemma 1.4] in the sense that if $j$ is linked to $l$ in $D$, then between two occurrences of a reflection $s_{j}$, there is one and only one occurrence of a reflection $s_{l}$. This excludes the possibility of horizontal adjacencies inside $H_{i}\left(\mathbf{w}_{0}\right)$. We have therefore $\beta_{k}=s_{i_{1}} \cdots s_{i_{k-1}}\left(\alpha_{i_{k}}\right), \beta_{k^{\prime}}=$ $s_{i_{1}} \cdots s_{i_{k^{\prime}-1}}\left(\alpha_{i_{k^{\prime}}}\right)$, with $\left(\alpha_{i_{k}}, \alpha_{i_{k^{\prime}}}\right)=-1$. One has $\left(\beta_{k}, \beta_{k^{\prime}}\right)=\left(\alpha_{i_{k}}, s_{i_{k}} s_{i_{k+1}} \cdots s_{i_{k^{\prime}-1}}\left(\alpha_{i_{k^{\prime}}}\right)\right)$ by invariance of the Cartan scalar product under action of $W$. Now $i_{k+1}, \ldots i_{k^{\prime}-1}$ are all different from $i_{k}$ and $i_{k^{\prime}}$, which implies that $\left(\alpha_{i_{k}}, s_{i_{k}} s_{i_{k+1}} \cdots s_{i_{k^{\prime}-1}}\left(\alpha_{i_{k^{\prime}}}\right)\right)=1$.

By [2, Lemma 2.11], $\left(\beta_{k}, \beta_{k^{\prime}}\right)=1$ means that there is a path inside $\Gamma_{Q}$ from $\left[\beta_{k}\right]$ to $\left[\beta_{k^{\prime}}\right]$. Now this path may have only one vertex on each of the adjacent levels $i_{k}$ and $i_{k}^{\prime}$, so it can cross them only once. In view of the mesh structure of $\Gamma_{Q}$, the only possibility is that this path is reduced to a single arrow $\left[\beta_{k}\right] \longrightarrow\left[\beta_{k^{\prime}}\right]$.

Proposition 5.4. One has $Z_{i}\left(\mathbf{w}_{0}\right)=\Psi\left(P_{i}(Q)\right)$. Furthermore, these sets, seen as nonoriented subgraphs respectively of $\Gamma_{Q}$ and $G^{\circ}\left(\mathbf{w}_{0}\right)$, are isomorphic.

Only the first part of the proposition needs to be proved, the second then follows, using Proposition 5.3. We shall need two results concerning the matrix $R=\left(r_{i, j}\right)$ of the Ringel form of $Q$. Let us define for $i \in I, \rho_{i}:=\sum_{k=1}^{n} r_{k, i} \omega_{k}$. The weight $\rho_{i}$ is such that its coordinates in the basis of fundamental weights are given by the $i$ th column of $R$. In a similar manner, let us put, corresponding to the $i$ th line of $R, \rho_{i}^{t}:=\sum_{k=1}^{n} r_{i, k} \omega_{k}$.

Lemma 5.5. The action of the Coxeter element on the vectors $\rho_{i}, i \in I$ is given by $c \rho_{i}=-\rho_{i}^{t}$.

Proof. Case-by-case analysis, following the four cases for the neighbourhood of $i$ in $Q$. Our convention is $\omega_{0}=\omega_{n+1}=0$.

Case 1. $i$ is a source of $Q$

We have in this case $\rho_{i}=\omega_{i}, \rho_{i}^{t}=-\omega_{i-1}+\omega_{i}-\omega_{i+1}$ and $c=s_{i} c_{1}, c_{1}$ being a product of simple reflections $s_{j}$ with $j \neq i$. As $s_{j}\left(\omega_{i}\right)=\omega_{i}$ for $j \neq i$, we see $c \rho_{i}=s_{i}\left(\omega_{i}\right)=$ $\omega_{i-1}-\omega_{i}+\omega_{i+1}$.

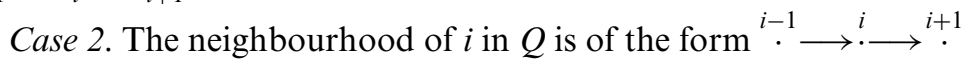

We have in this case $\rho_{i}=-\omega_{i-1}+\omega_{i}, \rho_{i}^{t}=\omega_{i}-\omega_{i+1}$ and $c=c_{1} s_{i} c_{2}$, where $c_{1}$ is a product of simple reflections $s_{j}$ with $j \geq i+1$, and $c_{2}$ is a product of simple reflections $s_{j}$ with $j \leq i-1$. We get

$$
\begin{aligned}
c_{1} \rho_{i} & =\rho_{i}, \\
s_{i} c_{1} \rho_{i} & =s_{i} \rho_{i}=-\omega_{i}+\omega_{i+1}, \\
c_{2} s_{i} c_{1} \rho_{i} & =-\omega_{i}+\omega_{i+1} .
\end{aligned}
$$

The other two cases follow by similar computations.

Recall we associated to a vertex $v_{\beta} \in G^{\circ}\left(\mathbf{w}_{0}\right)$, the weight $\lambda^{+}\left(v_{\beta}\right)$. Let $\varphi_{R}$ be the linear mapping of the Euclidean space $\mathbb{R} \Phi$ defined by $\varphi_{R}\left(\alpha_{i}\right)=-\rho_{i}, i \in I$. 
THEOREM 5.6 ([22, Theorem 2.4]).

$$
\forall \beta \in \Phi^{+}, \quad \lambda^{+}\left(v_{\beta}\right)=\varphi_{R}(\beta) .
$$

The key of the theorem is [22, Lemma 2.2]: If $k$ is a sink of an $A D E$ quiver $Q$, $Q^{\prime}:=s_{k} Q$, and $R, R^{\prime}$ are the respective Ringel matrices, then $s_{k} \varphi_{R^{\prime}}=\varphi_{R} s_{k}$. This lemma implies that $\varphi_{R}$ commutes with $c^{-1}$, and hence with $c=\left(c^{-1}\right)^{n}$. If $\beta \in \Phi^{+}$such that $c \beta \in \Phi^{+}$(that is, if $\tau$ is defined on $[\beta]$ ), then $c \lambda^{+}\left(v_{\beta}\right)=c \varphi_{R}(\beta)=\varphi_{R}(c \beta)$. We have then $c \lambda^{+}\left(v_{\beta}\right)=\lambda^{+}\left(v_{c \beta}\right)=\lambda^{-}\left(v_{\beta}\right)$.

Proof of Proposition 5.4. For any $\beta \in \Phi^{+}$, one has $\left(\beta, \alpha_{i}\right)_{R}=\left(\beta, \rho_{i}\right)$. This implies, by Theorem 3.1, that $[\beta] \in P_{i}(Q)$ if and only if $\left(\beta, \rho_{i}\right)>0$.

Let us apply a similar analysis for a vertex $v_{\beta}$ of $Z_{i}\left(\mathbf{w}_{0}\right)$. A chamber is in $\mathcal{Z}_{i}\left(\mathbf{w}_{0}\right)$ if the strand $L_{i}$ passes above it, and the strand $L_{i+1}$ passes below it. This means that the index $i$ appears in the labelling of the chamber and the index $i+1$ does not appear. Now the index $i$ corresponds to the weight $-\omega_{i-1}+\omega_{i}$ and the index $i+1$ to the weight $-\omega_{i}+\omega_{i+1}$. The condition above in terms of strands, translates in terms of weights, to having $\omega_{i}$ with coefficient 1 in $\lambda^{-}\left(v_{\beta}\right)$. Thus, $v_{\beta} \in Z_{i}\left(\mathbf{w}_{0}\right)$ if and only if $\left(\alpha_{i}, \lambda^{-}\left(v_{\beta}\right)\right)>0$.

There is only one projective indecomposable module in $P_{i}(Q)$, namely the projective cover of $S_{i}$, which is on the $i$ th translation level of $\Gamma_{Q}$. Likewise, the left border chamber of $\mathcal{W D}\left(\mathbf{w}_{0}\right)$ on level $j, j \in I$, has the weight $\omega_{j}$. Hence, the only vertex on the left border of $G^{0}\left(\mathbf{w}_{0}\right)$ verifying $\left(\alpha_{i}, \lambda^{-}\left(v_{\beta}\right)\right)>0$ is the one on the $i$ th level.

It remains to verify Proposition 5.4 for non-projective vertices of $\Gamma_{Q}$. Lemma 5.5 gives us for all $i, k$ that $\left(\alpha_{k}, \rho_{i}\right)=-\left(\alpha_{i}, c \rho_{k}\right)$. If $\beta=\sum_{k=1}^{n} m_{k} \alpha_{k}$, then

$$
\begin{aligned}
\left(\beta, \rho_{i}\right) & =\sum_{k=1}^{n} m_{k}\left(\alpha_{k}, \rho_{i}\right) \\
& =-\sum_{k=1}^{n} m_{k}\left(\alpha_{i}, c \rho_{k}\right) \\
& =\left(\alpha_{i}, c\left(-\sum_{k=1}^{n} m_{k} \rho_{k}\right)\right) \\
& =\left(\alpha_{i}, c \lambda^{+}\left(v_{\beta}\right)\right) \\
& =\left(\alpha_{i}, \lambda^{-}\left(v_{\beta}\right)\right) .
\end{aligned}
$$

We see that $\left(\beta, \rho_{i}\right)>0$ if and only if $\left(\alpha_{i}, \lambda^{-}\left(v_{\beta}\right)\right)>0$, so the first statement of the proposition is verified.

6. String cone inequalities and Lusztig moves. A vertex occurring in a GP-path of type $i$ must belong to a chamber of $\mathcal{Z}_{i}\left(\mathbf{w}_{0}\right)$. We shall denote by $Y_{i}\left(\mathbf{w}_{0}\right)$ the set of these vertices. We have already accounted for the set $Z_{i}\left(\mathbf{w}_{0}\right)$, which consists of rightmost vertices of the chambers in $\mathcal{Z}_{i}\left(\mathbf{w}_{0}\right)$. It remains to compute $Y_{i}\left(\mathbf{w}_{0}\right) \backslash Z_{i}\left(\mathbf{w}_{0}\right)$. As we shall now see, these vertices lie on the border path $\delta_{i}$.

Proposition 6.1. Fix $i \in I$. Then the subgraph with vertices $Y_{i}\left(\mathbf{w}_{0}\right)$ is given inside $G\left(\mathbf{w}_{0}, i\right)$, according to the neighbourhood of $i$ inside $Q$, by Figures 5 (with the relative position of $Z_{i}\left(\mathbf{w}_{0}\right)$ inside $Y_{i}\left(\mathbf{w}_{0}\right)$ delimited by segmented lines $)$ : 

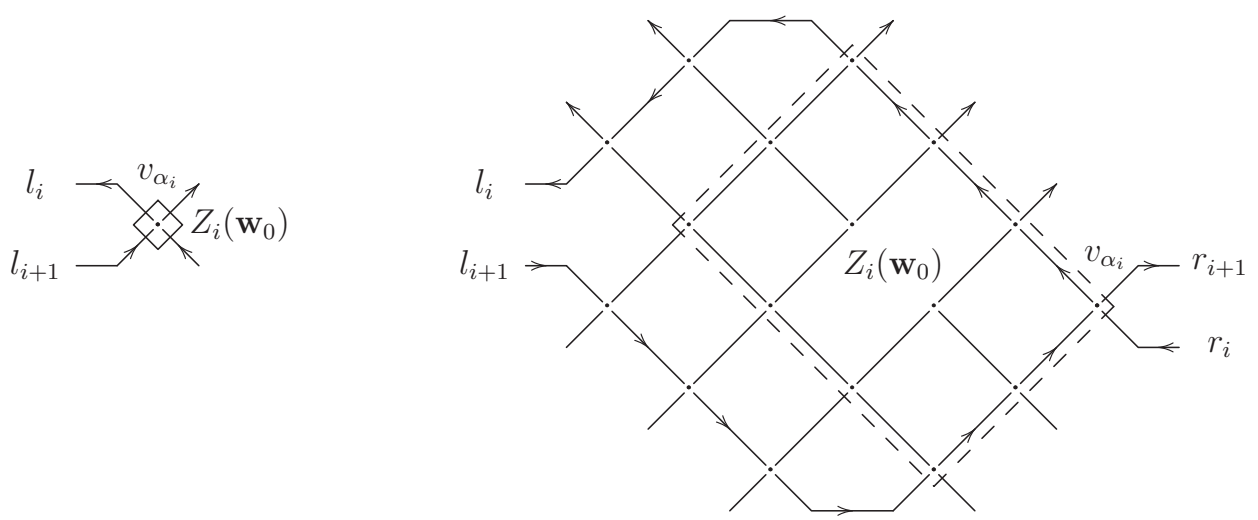

(a) $\stackrel{i-1}{\longrightarrow} \stackrel{i}{\longrightarrow} \longleftarrow^{i+1} \cdot \stackrel{\cdot}{ }$

(b) $\stackrel{i-1}{\longleftarrow} \stackrel{i}{\longrightarrow} \stackrel{i+1}{\longrightarrow}$
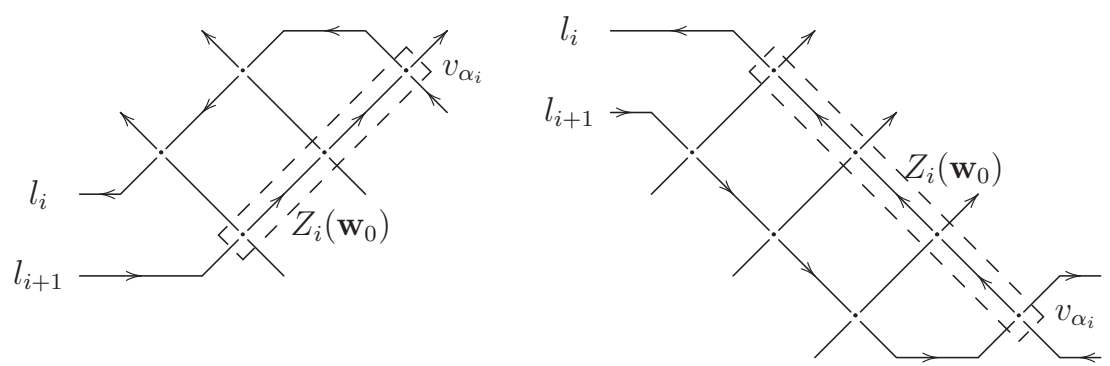

(c) $\stackrel{i-1}{\cdot} \longleftarrow^{i} \cdot \longleftarrow^{i+1} \cdot$

$(\mathrm{d}) \stackrel{i-1}{\longrightarrow} \stackrel{i}{\longrightarrow} \cdot \stackrel{i+1}{ } \cdot$

Figure 5. Structure of $Y_{i}\left(\mathbf{w}_{0}\right)$ inside $G\left(\mathbf{w}_{0}, i\right)$.

Proof.

The key to the proof is the combinatorics of $H_{i}(Q)$ (and therefore of $H_{i}\left(\mathbf{w}_{0}\right)$ ). Let $\left(j_{1}, j_{2} \cdots j_{i} \mid j_{i+1} \cdots j_{n+1}\right)$ be the $i$ th segmented expression of $c$. The vertex of $H_{i}(Q)$ at position $(k, l)$ in Figure 4 is mapped by $\Psi$ onto the intersection of the pseudolines $L_{j_{k}}$ and $L_{j_{l}}$, where $j_{k} \leq i$ and $j_{l} \geq i+1$. The structure of $Z_{i}\left(\mathbf{w}_{0}\right)$ inside $G\left(\mathbf{w}_{0}, i\right)$ then follows from Proposition 5.4. It remains to understand the position of the border path $\delta_{i}$ with respect to $Z_{i}\left(\mathbf{w}_{0}\right)$.

We shall call the set of vertices $v_{\beta} \in Z_{i}\left(\mathbf{w}_{0}\right)$ such that either $\tau_{w d}\left(v_{\beta}\right)$ is not defined or $\tau_{w d}\left(v_{\beta}\right) \notin Z_{i}\left(\mathbf{w}_{0}\right)$, the left border of $Z_{i}\left(\mathbf{w}_{0}\right)$. We shall call the set of vertices $v_{\beta} \in Z_{i}\left(\mathbf{w}_{0}\right)$, which are not the image under $\tau_{w d}$ of another vertex of $Z_{i}\left(\mathbf{w}_{0}\right)$, the right border of $Z_{i}\left(\mathbf{w}_{0}\right)$. We shall see that the limiting path $\delta_{i}$ consists of vertices either lying on the right border of $Z_{i}\left(\mathbf{w}_{0}\right)$ or obtained by applying the translation $\tau_{w d}$ on a vertex lying on the left border of $Z_{i}\left(\mathbf{w}_{0}\right)$. This ensures that vertices of $Y_{i}\left(\mathbf{w}_{0}\right)$ that do not belong to $Z_{i}\left(\mathbf{w}_{0}\right)$ lie on $\delta_{i}$.

Let us consider the part $\delta_{i}^{<}$of $\delta_{i}$ lying on the pseudoline $L_{i}$.

Case 1 . One has $\stackrel{i-1}{\longrightarrow} \longrightarrow \stackrel{i}{\longrightarrow}$ inside $Q$. 
The $i$-segmented expression of $c$ is such that $j_{i}=i$. In view of Proposition $5.2(\mathrm{~b}), L_{i}$ exits $Z_{i}\left(\mathbf{w}_{0}\right)$ on the leftmost vertex $v_{\beta_{\min }}$ of $H_{i}\left(\mathbf{w}_{0}\right)$. Now $\left[\beta_{\min }\right]$ is a projective isoclass of $\bmod \mathbb{C} Q$, hence $v_{\beta_{\text {min }}}$ is on the left border of $G^{\circ}\left(\mathbf{w}_{0}\right)$. The pseudoline $L_{i}$ goes directly to $l_{i}$ after leaving this vertex. The segment $\delta_{i}^{<}$contains only one vertex of $Z_{i}\left(\mathbf{w}_{0}\right)$, namely $v_{\beta_{\min }}$, which lies on the right border of this set.

Case 2. One has $\stackrel{i-1}{\cdot} \longleftarrow^{i}$. inside $Q$.

This time $j_{1}=i$, so $L_{i}$ leaves $Z_{i}\left(\mathbf{w}_{0}\right)$ on level 1 . Recall $j_{i+1}, \ldots j_{n+1}$ is a permutation of the interval $i+1, i+2, \ldots, n+1$, so $Z_{i}\left(\mathbf{w}_{0}\right)$ contains all vertices $L_{i} \cap L_{k}, k \geq i+1$, these vertices being on the right border of $Z_{i}\left(\mathbf{w}_{0}\right)$.

Let us fix $k \leq i-1$, and apply $\tau_{w d}$ on the leftmost vertex $L_{j_{k}} \cap L_{j_{n+1}}$ of $Z_{i}\left(\mathbf{w}_{0}\right)$ on level $k$. The result is, according to the $i$-segmented expression of $c$, the vertex $L_{j_{k+1}} \cap L_{j_{1}}$. Yet $j_{1}=i$, and $j_{2}, j_{3}, \ldots j_{i}$ is a permutation of $1,2, \ldots, i-1$. We see that all vertices $L_{i} \cap L_{k}$ with $k \leq i-1$ lie on $\delta_{i}^{<}$. We have accounted for all vertices lying on $L_{i}$ so that after leaving $Y_{i}\left(\mathbf{w}_{0}\right) L_{i}$ goes to $l_{i}$.

The case of the part $\delta_{i}^{>}$of $\delta_{i}$ lying on strand $L_{i+1}$ is totally symmetric, so all details are not given. The two cases to consider correspond to the orientations of the arrow between $i$ and $i+1$. If one has $\stackrel{i}{\cdot} \longleftarrow^{i+1}$ inside $Q, j_{n+1}=i+1$ so $L_{i+1}$ enters $Z_{i}\left(\mathbf{w}_{0}\right)$ on the first (and projective) vertex $v_{\beta_{\min }}$, directly from the border vertex $l_{i+1}$. In the other case, $\stackrel{i}{\longrightarrow} \stackrel{i+1}{\longrightarrow}$ inside $Q, L_{i+1}$ enters $Z_{i}\left(\mathbf{w}_{0}\right)$ on level $n$, and we can account for all vertices $L_{k} \cap L_{i+1}$. Either these lie on the right border of $Z_{i}\left(\mathbf{w}_{0}\right)$ if $k \leq i$, or are translates of vertices at levels $k=i+2, \ldots n$ of the left border of that set.

Proof of the Main Theorem 2.4. We shall proceed in two steps: First, we establish a one-to-one correspondence $\pi \mapsto A(\pi)$ between GP-paths of type $i$ and antichains of $P_{i}(Q)$, then fixing a GP-path $\pi$, we show that the vector $\mathbf{k}_{\pi}$ has the same coefficients as the Lusztig move $\mathbf{I}_{A(\pi)}$.

A GP-path $\pi$ of type $i$, by the oriented graph structure of $Y_{i}\left(\mathbf{w}_{0}\right)$ detailed in Figure 5, consists of three parts: A segment of $\delta_{i}^{>}$from $l_{i+1}$ up to entry into $Z_{i}\left(\mathbf{w}_{0}\right)$, a path $\pi_{\text {grid }}$ with vertices belonging to $Z_{i}\left(\mathbf{w}_{0}\right)$ up to an exit vertex, and finally returning to $l_{i}$ on a segment of $\delta_{i}^{<}$. The first and last segments are uniquely defined by the first and last vertices of $\pi_{\text {grid }}$, thus the segment $\pi_{\text {grid }}$ uniquely defines the whole path $\pi$.

Let us use the coordinate system given in Proposition 5.2 for $P_{i}(Q)$, which lies inside $H_{i}(Q)$. The order structure of $P_{i}(Q)$ is given by $\left(k_{1}, l_{1}\right) \leq\left(k_{2}, l_{2}\right)$ if and only if $k_{1} \geq k_{2}$ and $l_{1} \geq l_{2}$. All the vertices of $Z_{i}\left(\mathbf{w}_{0}\right)$ are by Propositions 5.2 and 5.4 of the same type: a crossing of a line $L_{j_{k}}$ with $j_{k} \leq i$, which points backwards and upwards, with a line $L_{j_{l}}$ with $j_{l} \geq i+1$, which points forwards and upwards. The path $\pi_{\text {grid }}$ is therefore a staircase path. As such, it is uniquely defined by the 'extremities' of its steps, vertices which are a turning point from a forward line into a backward line. The inverse image under $\Psi$ of these vertices inside $P_{i}(Q)$ form a set $\left\{\left(k_{1}, l_{1}\right),\left(k_{2}, l_{2}\right), \ldots,\left(k_{r}, l_{r}\right)\right\}$ whose elements have coordinates that are pairwise disjoint: for any $j \neq j^{\prime}$, one has $k_{j} \neq k_{j^{\prime}}$ and $l_{j} \neq l_{j^{\prime}}$. By the nature of the order structure of $P_{i}(Q)$, this set is an antichain that we shall denote by $A(\pi)$.

Conversely, an antichain of $P_{i}(Q)$ is a set of vertices $\left\{\left(k_{1}, l_{1}\right),\left(k_{2}, l_{2}\right), \ldots,\left(k_{r}, l_{r}\right)\right\}$ that have coordinates, which are pairwise disjoint. The images of these vertices under $\Psi$ form the extremities of a uniquely defined staircase path $\pi_{\text {grid }}$ of $Z_{i}\left(\mathbf{w}_{0}\right)$. By adding the appropriate segments of the limiting path $\delta_{i}$, we get a path $\pi(A)$ starting from $l_{i+1}$ and returning to $l_{i}$. The nature of crossings inside $Z_{i}\left(\mathbf{w}_{0}\right)$ and Lemma 4.2 exclude the existence of forbidden crossings. The path $\pi(A)$ is therefore a GP-path of type $i$. 
- Positive contribution

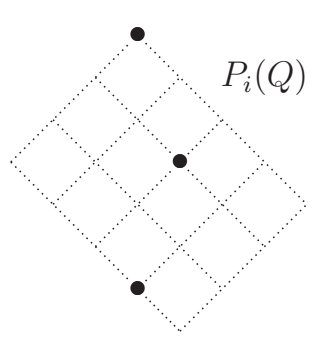

Antichain $A$

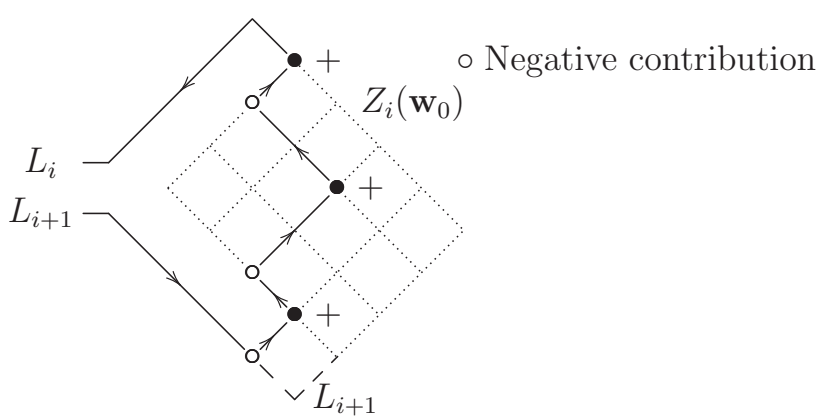

GP-path $\pi(A)$

Figure 6. Antichain of $P_{i}(Q)$ and corresponding GP-path of type $i$.

The mapping $A \mapsto \pi(A)$ is the inverse of $\pi \mapsto A(\pi)$ and vice-versa, so we have a one-to-one correspondence $\psi$ between GP-paths of type $i$ and antichains of $P_{i}(Q)$.

Let us now fix a GP-path and study its contributing vertices. Recall, a vertex $v_{\beta_{h}}$ contributes a term $+t_{h}$ in $\mathbf{k}_{\pi} \cdot \mathbf{t}$ if $\pi$ changes strands $L_{j} \longrightarrow L_{k}$ at $v_{\beta_{h}}$ with a decrease from $j$ to $k$, and a term $-t_{h}$ if a change of strands $L_{j} \longrightarrow L_{k}$ occurs at $v_{\beta_{h}}$ with an increase from $j$ to $k$. If $\pi$ stays on the same strand while passing through $v_{\beta_{h}}$, no contribution occurs.

In view of Figure 6, a change of strands occurs at an extremal vertex $v_{\beta_{h}}$ of $\pi_{\text {grid }}$. At this vertex, the incoming strand $L_{j_{k}}$ verifies $j_{k} \geq i+1$, while the outgoing strand $L_{j_{l}}$ verifies $j_{l} \leq i$. We see that a decrease of indices occurs in the passage $L_{j_{k}} \longrightarrow L_{j_{l}}$, so $v_{\beta_{h}}$ is a positively contributing vertex.

Let us denote by $J_{\pi}$ the order ideally defined by the extremal vertices of $\pi_{\text {grid }}$ inside $Z_{i}\left(\mathbf{w}_{0}\right)$ endowed with the poset structure induced by $P_{i}(Q)$. Consider a minimal element $v_{\beta_{h^{\prime}}}$ of $Z_{i}\left(\mathbf{w}_{0}\right) \backslash J_{\pi}$, and apply translation $\tau_{w d}$. We get a vertex $\tau_{w d}\left(v_{\beta_{h^{\prime}}}\right)$ of $\pi$ with the following possible cases (as illustrated by Figure 7):

(i) $\tau_{w d}\left(v_{\beta_{h^{\prime}}}\right)$ is in $Z_{i}\left(\mathbf{w}_{0}\right)$, in which case it is adjacent to three chambers of $\mathcal{Z}_{i}\left(\mathbf{w}_{0}\right)$. A passage from a backward-oriented line $L_{j_{k}}$ to a forward-oriented line $L_{j_{l}}$ occurs with an increase of indices.

(ii) $\tau_{w d}\left(v_{\beta_{h^{\prime}}}\right)$ is a vertex of $\delta_{i}^{>}$outside $Z_{i}\left(\mathbf{w}_{0}\right)$. The strand $L_{i+1}$ is of minimal index among forwardly oriented strands, so an increase of indices occurs by Proposition 6.1 .

(iii) $\tau_{w d}\left(v_{\beta_{h^{\prime}}}\right)$ is a vertex of $\delta_{i}^{<}$outside $Z_{i}\left(\mathbf{w}_{0}\right)$. The strand $L_{i}$ is of maximal index among backward-oriented strands, so again by Proposition 6.1 an increase of indices occurs.

We see in all cases, the vertex $\tau_{w d}\left(v_{\beta_{h^{\prime}}}\right)$ produces a negative contribution -1 to $\mathbf{k}_{\pi}$.

Finally, if $v_{\beta_{h}}$ is neither maximal inside $J_{\pi}$ nor a translate of a minimal element of $Z_{i}\left(\mathbf{w}_{0}\right) \backslash J_{\pi}$, then $\pi$ stays on the same strand while passing through it so that no contribution occurs. 

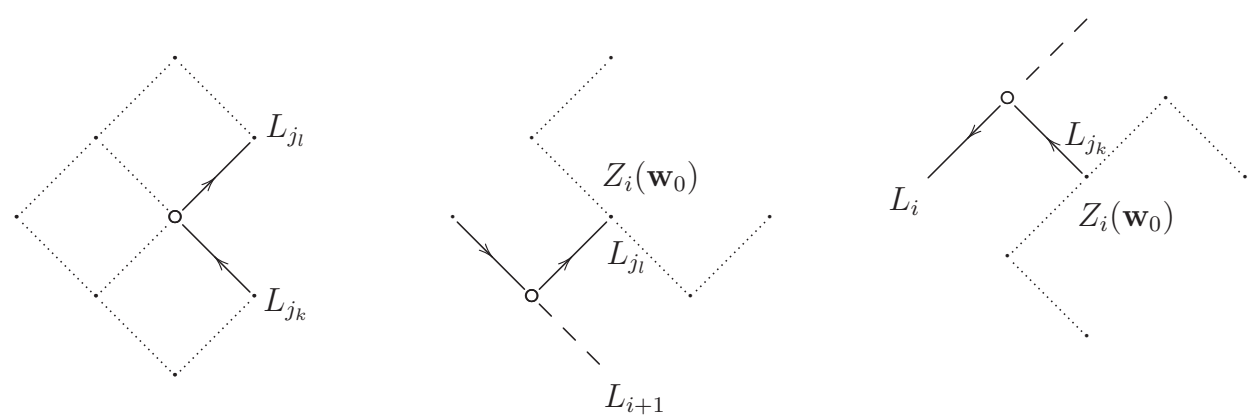

Figure 7. Negative contributing vertices.

Comparing this discussion with the definition of the Lusztig move out of the antichain $A(\pi)$, we see that we get exactly the same positive and negative contributions $\pm t_{h}$ in both cases.

REMARKS.

(i) The staircase paths used in the proof seem closely related to the Lediagrams defined by Postnikov [17] in his study of positroids (compare Figure 6 with [17, Figure 17.3]).

(ii) The neat structure of $Y_{i}\left(\mathbf{w}_{0}\right)$ described in Figure 5 breaks down for reduced expressions $\mathbf{w}_{0}$ of type $A_{n}$ that are not adapted to a quiver. Its subset $Z_{i}\left(\mathbf{w}_{0}\right)$ may include vertices that lie outside of $H_{i}\left(\mathbf{w}_{0}\right)$. Furthermore, the regular grid structure of $Z_{i}\left(\mathbf{w}_{0}\right)$ is destroyed by the appearance of horizontal adjacencies between vertices of $Z_{i}\left(\mathbf{w}_{0}\right)$.

7. A $D_{n}$ example. Let us consider the $D_{4}$-type quiver $Q$, given in the appendix of [18] as satisfying condition $(L)$ :

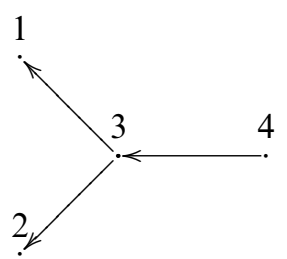

Let us verify the conjecture stated in Section 2. $\mathbf{w}_{0}=s_{1} s_{2} s_{3} s_{1} s_{2} s_{4} s_{3} s_{1} s_{2} s_{4} S_{3} S_{4}$ is adapted to $Q$, with reflection ordering

$$
\begin{array}{lll}
\beta_{1}=\alpha_{1} & \beta_{5}=\alpha_{1}+\alpha_{3} & \beta_{9}=\alpha_{2}+\alpha_{3}+\alpha_{4} \\
\beta_{2}=\alpha_{2} & \beta_{6}=\alpha_{1}+\alpha_{2}+\alpha_{3}+\alpha_{4} & \beta_{10}=\alpha_{3} \\
\beta_{3}=\alpha_{1}+\alpha_{2}+\alpha_{3} & \beta_{7}=\alpha_{1}+\alpha_{2}+2 \alpha_{3}+\alpha_{4} & \beta_{11}=\alpha_{3}+\alpha_{4} \\
\beta_{4}=\alpha_{2}+\alpha_{3} & \beta_{8}=\alpha_{1}+\alpha_{3}+\alpha_{4} & \beta_{12}=\alpha_{4}
\end{array}
$$

The Lusztig parametrization is given by elements $\mathbf{t} \in \mathbb{N}^{12}$ with $t_{i}$ corresponding to $\left[\beta_{i}\right]$. We shall concisely denote a vertex $[\beta]$ of $\Gamma_{Q}$ by the indexes of the appearing simple roots. For instance, 134 stands for $\left[\alpha_{1}+\alpha_{3}+\alpha_{4}\right]$. We shall denote the vertex 
$\left[\alpha_{1}+\alpha_{2}+2 \alpha_{3}+\alpha_{4}\right]$ by $12 \overline{3} 4$. The Auslander-Reiten quiver is given by

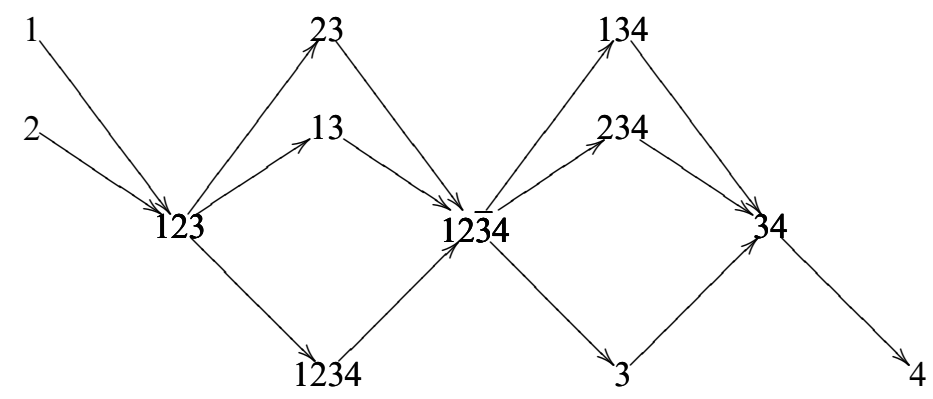

The $P_{i}(Q)$ sets are:

$$
\begin{aligned}
& P_{1}(Q)=\{1\} \\
& P_{2}(Q)=\{2\} \\
& P_{3}(Q)=\{123,23,13,12 \overline{3} 4,3\} \\
& P_{4}(Q)=\{1234,12 \overline{3} 4,134,234,34,4\}
\end{aligned}
$$

There are respectively 1, 1, 6, 7 antichains of types 1,2,3,4.

The Kashiwara embedding corresponds to [4, Theorem 5.10]. The computation of the set $K_{\mathbf{w}_{0}}^{B Z}$ requires therefore, by [4, Proposition 3.3 (iii)], the use of the reduced expression $\mathbf{w}_{0}^{o p}$, whose reflection ordering is reversed as compared with that of $\mathbf{w}_{0}\left(\alpha_{4}\right.$ occurs first, $\alpha_{1}$ occurs last), as well as a reversal in the numbering of coordinates. In our case, the set of indices of $\mathbf{w}_{0}^{o p}$ is $\mathbf{i}^{o p}=(4,3,4,2,1,3,4,2,1,3,2,1)$.

Fix $i \in I$, and let $E\left(\omega_{i}\right)$ be the corresponding fundamental representation of $U_{q}(\mathfrak{g})$ of type $D_{4}$. An $\mathbf{i}^{\text {op }}$-trail $\pi$ of type $i$ goes from $\omega_{i}$ to $w_{0} s_{i} \omega_{i}$. It is given by a set of coefficients $\mathbf{m}=\left(m_{1}, m_{2}, \ldots m_{12}\right)$ such that the monomial $e_{1}^{m_{1}} e_{2}^{m_{2}} \cdots e_{12}^{m_{12}}$ induces a nonzero mapping from the weight space $E\left(\omega_{i}\right)_{w_{0} s_{i} \omega_{i}}$ to the highest weight space $E\left(\omega_{i}\right)_{\omega_{i}}$. The path $\pi$ defines a sequence of weights $\omega_{i}=\gamma_{0}, \gamma_{1}, \ldots \gamma_{12}=w_{0} s_{i} \omega_{i}$ with

$$
\gamma_{k}:=\gamma_{0}-\sum_{l=1}^{k} m_{l} \alpha_{i_{l}}
$$

The trail $\pi$ defines a vector $\mathbf{h}_{\pi} \in \mathbb{Z}^{12}$ whose $k$ th coordinate is $h_{k}:=\left(\frac{\gamma_{k-1}+\gamma_{k}}{2}, \alpha_{i_{k}}\right)$, $(k=1, \ldots, 12)\left[4,(2.2)\right.$ p. 5]. Let us denote $\mathbf{k}_{\pi}:=\left(h_{12}, h_{11}, \ldots, h_{1}\right)$. Then by [4, Theorem 3.10], the set $K_{\mathbf{w}_{0}}^{B Z}$ of all vectors $\mathbf{k}_{\pi}$, where $\pi$ is any $\mathbf{i}^{\text {op }}$-trail, of any type $i$ defines $\mathcal{C}_{\mathrm{w}_{0}}$.

Let us consider the $\mathbf{i}^{o p}$-trail $\pi$ of type 3 with coefficients $(0,1,1,1,1,1,1,0,0$, $1,1,1)$. The list of weights $\gamma_{k}$ through which $\pi$ passes, as well as the coordinates $h_{k}$, are given by the following table (weights are expressed by their coordinates with respect to 
the basis of fundamental weights):

$\begin{array}{rlllr}k & \gamma_{k} & \left(\gamma_{k-1}+\gamma_{k}\right) / 2 & \alpha_{i_{k}} & h_{k} \\ 0 & {[0,0,1,0]} & & & \\ 1 & {[0,0,1,0]} & {[0,0,1,0]} & \alpha_{4} & 0 \\ 2 & {[1,1,-1,1]} & {\left[\frac{1}{2}, \frac{1}{2}, 0, \frac{1}{2}\right]} & \alpha_{3} & 0 \\ 3 & {[1,1,0,-1]} & {\left[1,1,-\frac{1}{2}, 0\right]} & \alpha_{4} & 0 \\ 4 & {[1,-1,1,-1]} & {\left[1,0, \frac{1}{2},-1\right]} & \alpha_{2} & 0 \\ 5 & {[-1,-1,2,-1]} & {\left[0,-1, \frac{3}{2},-1\right]} & \alpha_{1} & 0 \\ 6 & {[0,0,0,0]} & {\left[-\frac{1}{2},-\frac{1}{2}, 1,-\frac{1}{2}\right]} & \alpha_{3} & 1 \\ 7 & {[0,0,1,-2]} & {\left[0,0, \frac{1}{2},-1\right]} & \alpha_{4} & -1 \\ 8 & {[0,0,1,-2]} & {[0,0,1,-2]} & \alpha_{2} & 0 \\ 9 & {[0,0,1,-2]} & {[0,0,1,-2]} & \alpha_{1} & 0 \\ 10 & {[1,1,-1,-1]} & {\left[\frac{1}{2}, \frac{1}{2}, 0,-\frac{3}{2}\right]} & \alpha_{3} & 0 \\ 11 & {[1,-1,0,-1]} & {\left[1,0,-\frac{1}{2},-1\right]} & \alpha_{2} & 0 \\ 12 & {[-1,-1,1,-1]} & {\left[0,-1, \frac{1}{2},-1\right]} & \alpha_{1} & 0\end{array}$

We get $\mathbf{h}_{\pi}=(0,0,0,0,0,1,-1,0,0,0,0,0)$, hence $\mathbf{k}_{\pi}=(0,0,0,0,0,-1,1,0,0,0$, $0,0)$, which defines the string cone inequality $t_{7}-t_{6} \geq 0$. If we consider the antichain $A=\{12 \overline{3} 4\}$ of $P_{3}(Q)$, then $V_{A}=\left[\beta_{7}\right], U_{A}=\left[\beta_{6}\right]$, so we get $\mathbf{l}_{A}=\mathbf{k}_{\pi}$.

The enumeration of all possible $\mathbf{i}^{o p}$-trails in our case (with the help of quagroup package [7]) shows that there is a one-to-one correspondence between antichains $A$ of $P_{i}(Q)$ and $\mathbf{i}^{o p}$-trails $\pi$ of type $i$, for each of the types $i=1, \ldots, 4$. For every antichain $A$ there is a unique trail $\pi$ with $\mathbf{l}_{A}=\mathbf{k}_{\pi}$. We give these correspondences in the following table. The first column is the set of defining inequalities of $\mathcal{C}_{\mathbf{w}_{0}}$. Next to each inequality is the antichain $A$ defining the corresponding Lusztig move $\mathbf{l}_{A}$, and the $\mathbf{i}^{o p}$-trail leading to the corresponding vector $\mathbf{k}_{\pi} \in K_{\mathbf{w}_{0}}^{B Z}$.

\begin{tabular}{|c|c|c|c|c|}
\hline Type & Inequality & Antichain & $\mathbf{i}^{o p}$-trail position & $\mathbf{i}^{o p}$-trail coefficients $\mathbf{m}$ \\
\hline 1 & $t_{1} \geq 0$ & 1 & $43421 \underline{3} 4213 \underline{2} 1$ & $(0,0,0,0,1,1,1,1,0,1,0,0)$ \\
\hline 2 & $t_{2} \geq 0$ & 2 & $43421 \underline{3} 421 \underline{3} 21$ & $(0,0,0,1,0,1,1,0,1,1,0,0)$ \\
\hline \multirow[t]{6}{*}{3} & $t_{3}-t_{1}-t_{2} \geq 0$ & 123 & $4 \underline{3} \underline{42} 1 \underline{3} 4 \underline{2} 1321$ & $(0,1,1,1,1,2,1,1,1,0,0,0)$ \\
\hline & $t_{4}-t_{2} \geq 0$ & 23 & $4 \underline{3} \underline{4} \underline{2} \underline{3} \underline{4} \underline{2} 132 \underline{1}$ & $(0,1,1,1,1,2,1,1,0,0,0,1)$ \\
\hline & $t_{5}-t_{1} \geq 0$ & 13 & $4 \underline{3} \underline{4} \underline{1} \underline{3} \underline{4} 2 \underline{1} 3 \underline{2} 1$ & $(0,1,1,1,1,2,1,0,1,0,1,0)$ \\
\hline & $t_{4}+t_{5}-t_{3} \geq 0$ & 13,23 & $4 \underline{3} \underline{4} \underline{2} \underline{3} \underline{4} 213 \underline{2} 1$ & $(0,1,1,1,1,2,1,0,0,0,1,1)$ \\
\hline & $t_{7}-t_{6} \geq 0$ & $12 \overline{3} 4$ & $4 \underline{3} \underline{4} \underline{2} 1 \underline{3} 4213 \underline{2} 1$ & $(0,1,1,1,1,1,1,0,0,1,1,1)$ \\
\hline & $t_{10} \geq 0$ & 3 & $4 \underline{3} 4 \underline{2} \underline{3} \underline{4} 21 \underline{3} \underline{2} 1$ & $(0,1,0,1,1,1,2,0,0,1,1,1)$ \\
\hline \multirow[t]{7}{*}{4} & $t_{6}-t_{3} \geq 0$ & 1234 & $\underline{4} \underline{3} 42 \underline{2} \underline{3} 421321$ & $(1,1,0,1,1,1,0,0,0,0,0,0)$ \\
\hline & $t_{7}-t_{4}-t_{5} \geq 0$ & $12 \overline{3} 4$ & $\underline{4} \underline{3} 4 \underline{2} 13421321$ & $(1,1,0,1,1,0,0,0,0,1,0,0)$ \\
\hline & $t_{8}-t_{5} \geq 0$ & 134 & $\underline{4} \underline{3} 4 \underline{2} 1342 \underline{1} \underline{3} 21$ & $(1,1,0,1,0,0,0,0,1,1,0,0)$ \\
\hline & $t_{9}-t_{4} \geq 0$ & 234 & $\underline{4} \underline{3} 42 \underline{1} 34 \underline{2} 1 \underline{3} 21$ & $(1,1,0,0,1,0,0,1,0,1,0,0)$ \\
\hline & $t_{8}+t_{9}-t_{7} \geq 0$ & 134,234 & $\underline{4} \underline{3} 42134 \underline{2} 1 \underline{3} 21$ & $(1,1,0,0,0,0,0,1,1,1,0,0)$ \\
\hline & $t_{11}-t_{10} \geq 0$ & 34 & $\underline{4} 3421 \underline{3} 4 \underline{2} \underline{1} \underline{3} 21$ & $(1,0,0,0,0,1,0,1,1,1,0,0)$ \\
\hline & $t_{12} \geq 0$ & 4 & $43 \underline{4} 21 \underline{3} 4 \underline{2} 1 \underline{3} 21$ & $(0,0,1,0,0,1,0,1,1,1,0,0)$ \\
\hline
\end{tabular}

REMARK. The trail $\pi$ of type 3 considered above in detail passes through the weight $[0,0,0,0]$. All other trails of the table pass only through extremal weights. The existence of $\pi$ shows that condition $(L)$ is not strong enough to allow an analogue of [4, Theorem 3.14]. The subexpression corresponding to $\pi$ is not a reduced word. 


\section{REFERENCES}

1. M. Auslander, I. Reiten and S. O. Smalo, Representation theory of Artin algebras, Cambridge Studies in Advanced Mathematics, no. 36 (Cambridge University Press, Cambridge, UK, 1995).

2. R. Bedard, On commutation classes of reduced words in Weyl groups, Eur. J. Comb. 20 (1999), 483-505.

3. A. Berenstein and A. Zelevinsky, String bases for quantum groups of type $A_{r} ;$ I. M. Gelfand Seminar, Adv. Soviet Math. 16 (part 1) (1993), 51-89.

4. A. Berenstein and A. Zelevinsky, Tensor product multiplicities, canonical bases, and totally positive varieties, Invent. Math. 143 (2001), 77-128.

5. I. N. Bernstein, I. M. Gelfand and V. A. Ponomarev, Coxeter functors and Gabriel's theorem, Russ. Math. Surv. 28 (1973), 17-32.

6. S. Brenner, A combinatorial characterisation of finite Auslander-Reiten quivers, in Representation theory I. Finite dimensional algebras (Dlab V., Gabriel P., Michler G., Editor), Springer LNM 1177, (Springer, New York, 1986), 13-49.

7. W. De Graaf, Quagroup: A GAP4 package for doing computations with quantum groups (2003), available at http://www.science.unitn.it/degraaf/quagroup.html.

8. The GAP Group, GAP - groups, algorithms, and programming, version 4.4.12, 2008, available at http://www.gap-system.org

9. $\mathrm{P}$. Gabriel, Auslander-Reiten sequences and representation-finite algebras; in Representation theory I, Proceedings of Workshop, Carleton University, Ottawa, Canada, 1979, Springer Lecture Notes in Mathematics 831 (Springer, Berlin, Germany, 1980), 1-71.

10. O. Gleizer and A. Postnikov, Littlewood-Richardson coefficients via Yang-Baxter equation, IMRN 14 (2000), 741-774.

11. A. Joseph, Quantum groups and their primitive ideals (Springer, Berlin, Germany, 1993).

12. M. Kashiwara, On crystal bases of the $q$-analogue of universal enveloping algebras, Duke Math. J. 63 (1991), 465-516.

13. M. Kashiwara, The crystal base and Littelmann's refined Demazure character formula, Duke Math. J. 71 (1993), 839-858.

14. P. Littelmann, Cones, crystals and patterns, Transf. Gr. 3 (1998), 145-179.

15. G. Lusztig, Canonical bases arising from quantized enveloping algebras, J. Am. Math. Soc. 3 (1990), 447-498.

16. G. Lusztig, Introduction to quantum groups, Progress in Mathematics, no. 110 (Birkhauser, Boston, MA, 1993).

17. A. Postnikov, Total positivity, Grassmannians, and networks, Preprint, available at http://www-math.mit.edu/ apost/papers/tpgrass.pdf, 2006.

18. M. Reineke, On the coloured graph structure of Lusztig's canonical basis, Math. Ann. 307 (1997), 705-723.

19. C. M. Ringel, Hall algebras, in Topics in Algebra, 26 (Banach Center, Poland, 1990), 433-447.

20. C. M. Ringel, Hall algebras and quantum groups, Invent. Math. 101 (1990), 583-592.

21. C. M. Ringel, PBW-bases of quantum groups, J. Reine Angew. Math. 470 (1996), 51-88. 35-55.

22. S. Zelikson, Auslander-Reiten quivers and the Coxeter complex, Alg. Rep. Th. 8 (2005), 